\title{
Finitely based monoids
}

\author{
Olga Sapir
}

\begin{abstract}
We present a method for proving that a semigroup is finitely based and find some new sufficient conditions under which a monoid is finitely based. As an application, we find a class of finite aperiodic monoids where the finite basis property behaves in a complicated way with respect to the lattice operations but can be recognized by a simple algorithm.

The method results in a short proof of the theorem of E. Lee that every monoid that satisfies $x t_{1} x y t_{2} y \approx x t_{1} y x t_{2} y$ and $x y t_{1} x t_{2} y \approx y x t_{1} x t_{2} y$ is finitely based. Also, the method gives an alternative proof of the theorem of $F$. Blanchet-Sadri that a pseudovariety of $n$-testable languages is finitely based if and only if $n \leq 3$.
\end{abstract}

Keywords: Finite Basis Problem, Semigroups, Monoids, piecewise-testable languages

\section{Introduction}

A set of identities $\Sigma$ is said to be finitely based if all identities in $\Sigma$ can be derived from a finite subset of $\Sigma$. The equational theory $(E q(S))$ of a semigroup $S$ is the set of all identities holding in $S$. A semigroup is said to be finitely based (FB) if its equational theory is finitely based. Otherwise, a semigroup is said to be non-finitely based (NFB). The following construction is attributed to Dilworth and was used by P. Perkins [10] to construct one of the first examples of finite NFB semigroups.

Let $\mathfrak{A}$ be an alphabet and $W$ be a set of words in the free monoid $\mathfrak{A}^{*}$. Let $S(W)$ denote the Rees quotient over the ideal of $\mathfrak{A}^{*}$ consisting of all words that are not subwords of words in $W$. For each set of words $W$, the semigroup $S(W)$ is a monoid with zero whose nonzero elements are the subwords of words in $W$. Evidently, $S(W)$ is finite if and only if $W$ is finite.

This article is the second part of a sequence of four submissions. The previous article [13] contains a method for proving that a semigroup is non-finitely based. In articles [14, 15] we study the following problem.

Question 1. [16, M. Sapir] Is the set of finite finitely based monoids of the form $S(W)$ recursive? 
If a variable $t$ occurs exactly once in a word $\mathbf{u}$ then we say that $t$ is linear in $\mathbf{u}$. If a variable $x$ occurs more than once in a word $\mathbf{u}$ then we say that $x$ is non-linear in $\mathbf{u}$. In article [14, we show how to recognize FB semigroups among the monoids of the form $S(W)$ where $W$ consists of a single word with at most two non-linear variables. In article [15], we show how to recognize FB semigroups among the monoids of the form $S(W)$ with some other natural restrictions on the set $W$.

We use var $\Delta$ to denote the variety defined by a set of identities $\Delta$ and $\operatorname{var} S$ to denote the variety generated by a semigroup $S$. The identities $x t_{1} x y t_{2} y \approx x t_{1} y x t_{2} y$, $x y t_{1} x t_{2} y \approx y x t_{1} x t_{2} y$ and $x t_{1} y t_{2} x y \approx x t_{1} y t_{2} y x$ we denote respectively by $\sigma_{\mu}, \sigma_{1}$ and $\sigma_{2}$. Notice that the identities $\sigma_{1}$ and $\sigma_{2}$ are dual to each other.

It follows from [14 that if $W$ consists of a single word with at most two non-linear variables and the monoid $S(W)$ is finitely based then $S(W)$ is contained either in $\operatorname{var}\left\{\sigma_{\mu}, \sigma_{1}\right\}$ or in $\operatorname{var}\left\{\sigma_{\mu}, \sigma_{2}\right\}$ or in $\operatorname{var}\left\{\sigma_{1}, \sigma_{2}\right\}$.

In [6], M. Jackson proved that $\operatorname{var} S\left(\left\{a t_{1} a b t_{2} b\right\}\right)$ and $\operatorname{var} S\left(\left\{a b t_{1} a t_{2} b, a t_{1} b t_{2} a b\right\}\right)$ are limit varieties in the sense that each of these varieties is NFB while each proper monoid subvariety of each of these varieties is FB. In order to determine whether $\operatorname{var} S\left(\left\{a t_{1} a b t_{2} b\right\}\right)$ and $\operatorname{var} S\left(\left\{a b t_{1} a t_{2} b, a t_{1} b t_{2} a b\right\}\right)$ are the only limit varieties generated by finite aperiodic monoids with central idempotents, he suggested in [6] to investigate the monoid subvarieties of $\operatorname{var}\left\{\sigma_{\mu}, \sigma_{1}\right\}$ and dually, of $\operatorname{var}\left\{\sigma_{\mu}, \sigma_{2}\right\}$. In [8], E. Lee proved that all finite aperiodic monoids with central idempotents contained in $\operatorname{var}\left\{\sigma_{\mu}, \sigma_{1}\right\}$ are finitely based. This result implies the affirmative answer to the question of Jackson posed in [6]. Later in [9], E. Lee proved that all monoids contained in $\operatorname{var}\left\{\sigma_{\mu}, \sigma_{1}\right\}$ are finitely based. This more general result implies that $\operatorname{var} S\left(\left\{a t_{1} a b t_{2} b\right\}\right)$ and $\operatorname{var} S\left(\left\{a b t_{1} a t_{2} b, a t_{1} b t_{2} a b\right\}\right)$ are the only limit varieties generated by aperiodic monoids with central idempotents.

In this article we present a method (see Lemma 3.1 below) that can be used for proving that a semigroup is finitely based. In sections 4 and 5 we use Lemma 3.1 to find some sufficient conditions under which a monoid in $\operatorname{var}\left\{\sigma_{1}, \sigma_{2}\right\}$ and in $\operatorname{var}\left\{\sigma_{\mu}\right\}$ is finitely based.

Lemma 3.1 allows to give a short proof of the result of Lee that every monoid contained in $\operatorname{var}\left\{\sigma_{\mu}, \sigma_{1}\right\}$ and in $\operatorname{var}\left\{\sigma_{\mu}, \sigma_{2}\right\}$ is finitely based (see Theorem 3.5 below). In contrast with the result of Lee, the finite basis property behaves in a complicated way in $\operatorname{var}\left\{\sigma_{1}, \sigma_{2}\right\}$. In particular, it is already complicated in the class of monoids of the form $A_{0}^{1} \times S(W)$ where $A_{0}^{1}$ is the monoid obtained by adjoining an identity element to the semigroup $A_{0}=\langle a, b \mid a a=a, b b=b, a b=0\rangle$ of order four and $S(W)$ is contained in $\operatorname{var}\left\{\sigma_{1}, \sigma_{2}\right\}$ (See Example 7.4 in [13] and Example 4.9 below). Nevertheless, Theorem 4.8 contains a simple algorithm for selecting finitely based monoids in this class.

In section 6 , we give a simple description of the equational theories for each of the seven monoid varieties defined by the subsets of $\left\{\sigma_{\mu}, \sigma_{1}, \sigma_{2}\right\}$. We also show that the monoid varieties defined by $\left\{\sigma_{\mu}, \sigma_{1}, \sigma_{2}\right\}$ and by $\left\{\sigma_{1}, \sigma_{2}\right\}$ are generated by monoids of the form $S(W)$.

Surprisingly, Lemma 3.1 works not only for monoids satisfying one of the identities in $\left\{\sigma_{\mu}, \sigma_{1}, \sigma_{2}\right\}$. In section 7 , we use Lemma 3.1 to reprove the result of $\mathrm{F}$. 
Blanchet-Sadri [2] that the equational theory $J_{3}$ (see the next section) of the monoid $S_{4}$ of all reflexive binary relations on a four-element set is finitely based.

\section{Preliminaries}

Throughout this article, elements of a countable alphabet $\mathfrak{A}$ are called variables and elements of the free semigroup $\mathfrak{A}^{+}$are called words. If $\mathfrak{X}$ is a set of variables then we write $\mathbf{u}(\mathfrak{X})$ to refer to the word obtained from $\mathbf{u}$ by deleting all occurrences of all variables that are not in $\mathfrak{X}$ and say that the word $\mathbf{u}$ deletes to the word $\mathbf{u}(\mathfrak{X})$. If $\mathfrak{X}=\left\{y_{1}, \ldots, y_{k}\right\} \cup \mathfrak{Y}$ for some variables $y_{1}, \ldots, y_{k}$ and a set of variables $\mathfrak{Y}$ then instead of $\mathbf{u}\left(\left\{y_{1}, \ldots, y_{k}\right\} \cup \mathfrak{Y}\right)$ we simply write $\mathbf{u}\left(y_{1}, \ldots, y_{k}, \mathfrak{Y}\right)$.

We say that a set of identities $\Sigma$ is closed under deleting variables, if for each set of variables $\mathfrak{X}$, the set $\Sigma$ contains the identity $\mathbf{u}(\mathfrak{X}) \approx \mathbf{v}(\mathfrak{X})$ whenever $\Sigma$ contains an identity $\mathbf{u} \approx \mathbf{v}$. We use $\Sigma^{\delta}$ to denote the closure of $\Sigma$ under deleting variables. For example, $\left\{\sigma_{\mu}\right\}^{\delta}=\left\{x t_{1} x y t_{2} y \approx x t_{1} y x t_{2} y, x x y t_{2} y \approx x y t_{2} y, x t_{1} x y y \approx\right.$ $\left.x t_{1} y x y, x x y y \approx x y x y\right\}$. If a semigroup $S$ satisfies all identities in a set $\Sigma$ then we write $S \models \Sigma$. If $S$ is a monoid then evidently, $S \models \Sigma$ if and only if $S \models \Sigma^{\delta}$.

A word $\mathbf{u}$ is said to be an isoterm [10] for a semigroup $S$ if $S$ does not satisfy any nontrivial identity of the form $\mathbf{u} \approx \mathbf{v}$. A word that contains at most one non-linear variable is called almost-linear. An identity $\mathbf{u} \approx \mathbf{v}$ is called almost-linear if both words $\mathbf{u}$ and $\mathbf{v}$ are almost-linear. The set $\operatorname{Cont}(\mathbf{u})=\left\{x \in \mathfrak{A} \mid \operatorname{occ}_{\mathbf{u}}(x)>0\right\}$ of all variables contained in a word $\mathbf{u}$ is called the content of $\mathbf{u}$. An identity $\mathbf{u} \approx \mathbf{v}$ is called regular if $\operatorname{Cont}(\mathbf{u})=\operatorname{Cont}(\mathbf{v})$.

Fact 2.1. If the word $x y$ is not an isoterm for a monoid $S$ and $S \models \sigma_{\mu}$ then $S$ is either finitely based by some almost-linear identities or $S \models x \approx x^{n}$ for some $n>1$ and satisfies only regular identities.

Proof. If $S$ satisfies an irregular identity then $S$ is a group with period $n>0$. Since $S$ satisfies the identity $x x y y \approx x y x y$, the group $S$ is finitely based by $\left\{y \approx x^{n} y \approx\right.$ $\left.y x^{n}, x y \approx y x\right\}$. So, we may assume that $S$ satisfies only regular identities.

Since the word $x y$ is not an isoterm for $S$, the monoid $S$ satisfies a non-trivial identity of the form $x y \approx \mathbf{u}$. Since $S$ satisfies only regular identities, we have that $\operatorname{Cont}(\mathbf{u})=\{x, y\}$. If the length of the word $\mathbf{u}$ is 2 then $S$ is commutative and is finitely based by either $\left\{x^{m} \approx x, x y \approx y x\right\}$ for some $m>1$ or by $x y \approx y x$. If the length of the word $\mathbf{u}$ is at least 3 then $S$ satisfies an identity $x \approx x^{n}$ for some $n>1$.

Lemma 2.2. [17, Corollary 2] Every set of almost-linear identities is finitely based.

Lemma 2.3. [9, Proposition 5.7] Every set of identities that consists of $\left\{\sigma_{1}, \sigma_{\mu}\right\}^{\delta}$ and some identities of the form

$x^{\alpha_{1}} y^{\beta_{1}} t_{1} x^{\alpha_{2}} y^{\beta_{2}} t_{2} \ldots t_{n-1} x^{\alpha_{n}} y^{\beta_{n}} t_{n} x^{\alpha_{n+1}} y^{\beta_{n+1}} \approx x^{\alpha_{1}} y^{\beta_{1}} t_{1} x^{\alpha_{2}} y^{\beta_{2}} t_{2} \ldots t_{n-1} x^{\alpha_{n}} y^{\beta_{n}} t_{n} y^{\beta_{n+1}} x^{\alpha_{n+1}}$ 
where $n, \alpha_{1}, \beta_{1}, \ldots, \alpha_{n}, \beta_{n} \geq 0$ and $\alpha_{n+1}, \beta_{n+1}>0$, is finitely based.

We use ${ }_{i \mathbf{u}} x$ to refer to the $i^{\text {th }}$ from the left occurrence of $x$ in $\mathbf{u}$. We use lastu $x$ to refer to the last occurrence of $x$ in $\mathbf{u}$. The set $\operatorname{OccSet}(\mathbf{u})=\left\{{ }_{i \mathbf{u}} x \mid x \in \mathfrak{A}, 1 \leq\right.$ $\left.i \leq \operatorname{occ}_{\mathbf{u}}(x)\right\}$ of all occurrences of all variables in $\mathbf{u}$ is called the occurrence set of $\mathbf{u}$. The word $\mathbf{u}$ induces a (total) order $<_{\mathbf{u}}$ on the $\operatorname{set} \operatorname{OccSet}(\mathbf{u})$ defined by ${ }_{i \mathbf{u}} x<_{\mathbf{u}}{ }_{j \mathbf{u}} y$ if and only if the $i^{\text {th }}$ occurrence of $x$ precedes the $j^{\text {th }}$ occurrence of $y$ in $\mathbf{u}$. If a pair $\{c, d\} \subseteq \operatorname{OccSet}(\mathbf{u})$ is adjacent in $\mathbf{u}$ and $c<_{\mathbf{u}} d$ then we write $c \ll_{\mathbf{u}} d$.

An identity $\mathbf{u} \approx \mathbf{v}$ is called balanced if for each variable $x \in \mathfrak{A}$ we have $\mathbf{u}(x)=$ $\mathbf{v}(x)$. If $\mathbf{u} \approx \mathbf{v}$ is a balanced identity then for each $x \in \mathfrak{A}$ and $1 \leq i \leq \operatorname{occ}_{\mathbf{u}}(x)=$ $\operatorname{occ}_{\mathbf{v}}(x)$ we identify ${ }_{i \mathbf{u}} x \in \operatorname{OccSet}(\mathbf{u})$ and ${ }_{i \mathbf{v}} x \in \operatorname{OccSet}(\mathbf{v})$. We say that a pair $\{c, d\} \subseteq \operatorname{OccSet}(\mathbf{u})$ is unstable in a balanced identity $\mathbf{u} \approx \mathbf{v}$ if $c<_{\mathbf{u}} d$ but $d<_{\mathbf{v}} c$. We say that a pair $\{c, d\} \subseteq \operatorname{OccSet}(\mathbf{u})$ is critical in a balanced identity $\mathbf{u} \approx \mathbf{v}$ if $\{c, d\}$ is adjacent in $\mathbf{u}$ and unstable in $\mathbf{u} \approx \mathbf{v}$. The next statement says that every non-trivial balanced identity contains a critical pair.

Lemma 2.4. [12, Lemma 3.2] If $\{c, d\} \subseteq \operatorname{OccSet}(\mathbf{u})$ is unstable in a balanced identity $\mathbf{u} \approx \mathbf{v}$ and $c<_{\mathbf{u}} d$ then for some $\{p, q\} \subseteq \operatorname{OccSet}(\mathbf{u})$ we have that $c \leq_{\mathbf{u}} p \ll_{\mathbf{u}}$ $q \leq_{\mathbf{u}} d$ and $\{p, q\}$ is also unstable in $\mathbf{u} \approx \mathbf{v}$.

We say that a word $\mathbf{u}=x_{1} x_{2} \ldots x_{k}$ is a scattered subword of a word $\mathbf{v}$ whenever there exist words $\mathbf{v}_{0}, \mathbf{v}_{1}, \ldots, \mathbf{v}_{k-1}, \mathbf{v}_{k} \in \mathfrak{A}^{*}$ such that $\mathbf{v}=\mathbf{v}_{0} x_{1} \mathbf{v}_{1} x_{2} \mathbf{v}_{2} \ldots \mathbf{v}_{k-1} x_{k} \mathbf{v}_{k}$; in other terms, this means that one can extract $\mathbf{u}$ treated as a sequence of letters $x_{1}, x_{2}, \ldots, x_{k}$ from the sequence $\mathbf{v}$.

We denote by $J_{m}$ the set of all identities $(\mathbf{u} \approx \mathbf{v})$ such that the words $\mathbf{u}$ and $\mathbf{v}$ have the same set of scattered subwords of length $\leq m$. For each $n>1$ we use $S_{n}$ to denote the monoid of all reflexive binary relations on a set with $n$ elements. In [18], M. Volkov proved that for each $m>0, J_{m}$ is the equational theory of $S_{m+1}$ and of several other interesting monoids (see also [1]). In view of the famous Eilenberg correspondence ([5], see also [11]), Theorem 2 in [18] says that for each $m>0$ the monoid $S_{m+1}$ generates the pseudovariety of piecewise $m$-testable languages.

\section{A method for proving that a semigroup is finitely based}

We use $\Sigma \vdash \Delta$ to indicate that every identity in $\Delta$ can be derived from some identities in $\Sigma$. The cardinality of a set $X$ is denoted by $|X|$.

Lemma 3.1. Let $\Sigma$ be a set of identities.

Let $\mathcal{P}$ and $\mathcal{Q}$ be some properties of identities such that the property $\mathcal{Q}$ is stronger than $\mathcal{P}$. Let Dist $(\mathcal{P} \rightarrow \mathcal{Q})$ be a function which associates with each $\mathcal{P}$-identity $\mathbf{u} \approx \mathbf{v}$ a set $\operatorname{Dist}(\mathcal{P} \rightarrow \mathcal{Q})(\mathbf{u} \approx \mathbf{v})$ so that the $\operatorname{set} \operatorname{Dist}(\mathcal{P} \rightarrow \mathcal{Q})(\mathbf{u} \approx \mathbf{v})$ is empty if and only if $\mathbf{u} \approx \mathbf{v}$ is a $\mathcal{Q}$-identity. 
Suppose that for each $\mathcal{P}$-identity $\mathbf{u} \approx \mathbf{v}$ which is not a $\mathcal{Q}$-identity, one can find a $\mathcal{P}$-identity $\mathbf{u}_{\mathbf{1}} \approx \mathbf{v}_{\mathbf{1}}$ such that $\Sigma \vdash\left\{\mathbf{u} \approx \mathbf{u}_{\mathbf{1}}, \mathbf{v} \approx \mathbf{v}_{\mathbf{1}}\right\}$ and $\mid \operatorname{Dist}(\mathcal{P} \rightarrow \mathcal{Q})\left(\mathbf{u}_{\mathbf{1}} \approx\right.$ $\left.\mathbf{v}_{\mathbf{1}}\right)|<| \operatorname{Dist}(\mathcal{P} \rightarrow \mathcal{Q})(\mathbf{u} \approx \mathbf{v}) \mid$

Then every $\mathcal{P}$-identity can be derived from $\Sigma$ and from a $\mathcal{Q}$-identity.

Proof. Let $\mathbf{u} \approx \mathbf{v}$ be an arbitrary $\mathcal{P}$-identity. If $\mathbf{u} \approx \mathbf{v}$ is not a $\mathcal{Q}$-identity then the set $\operatorname{Dist}(\mathcal{P} \rightarrow \mathcal{Q})(\mathbf{u} \approx \mathbf{v})$ is not empty. By our assumption, one can find a $\mathcal{P}$-identity $\mathbf{u}_{\mathbf{1}} \approx \mathbf{v}_{\mathbf{1}}$ such that $\Sigma \vdash\left\{\mathbf{u} \approx \mathbf{u}_{\mathbf{1}}, \mathbf{v} \approx \mathbf{v}_{\mathbf{1}}\right\}$ and $\mid \operatorname{Dist}(\mathcal{P} \rightarrow \mathcal{Q})\left(\mathbf{u}_{\mathbf{1}} \approx\right.$ $\left.\mathbf{v}_{\mathbf{1}}\right)|<| \operatorname{Dist}(\mathcal{P} \rightarrow \mathcal{Q})(\mathbf{u} \approx \mathbf{v}) \mid$

If the set $\operatorname{Dist}(\mathcal{P} \rightarrow \mathcal{Q})\left(\mathbf{u}_{\mathbf{1}} \approx \mathbf{v}_{\mathbf{1}}\right)$ is empty then we are done. Otherwise, by our assumption, one can find a $\mathcal{P}$-identity $\mathbf{u}_{\mathbf{2}} \approx \mathbf{v}_{\mathbf{2}}$ such that $\Sigma \vdash\left\{\mathbf{u}_{\mathbf{1}} \approx \mathbf{u}_{\mathbf{2}}, \mathbf{v}_{\mathbf{1}} \approx \mathbf{v}_{\mathbf{2}}\right\}$ and $\left|\operatorname{Dist}(\mathcal{P} \rightarrow \mathcal{Q})\left(\mathbf{u}_{\mathbf{2}} \approx \mathbf{v}_{\mathbf{2}}\right)\right|<\left|\operatorname{Dist}(\mathcal{P} \rightarrow \mathcal{Q})\left(\mathbf{u}_{\mathbf{1}} \approx \mathbf{v}_{\mathbf{1}}\right)\right|$.

By repeating this procedure $k \leq|\operatorname{Dist}(\mathcal{P} \rightarrow \mathcal{Q})(\mathbf{u} \approx \mathbf{v})|$ times, we obtain a $\mathcal{P}$-identity $\mathbf{u}_{\mathbf{k}} \approx \mathbf{v}_{\mathbf{k}}$ such that the $\operatorname{set} \operatorname{Dist}(\mathcal{P} \rightarrow \mathcal{Q})\left(\mathbf{u}_{\mathbf{k}} \approx \mathbf{v}_{\mathbf{k}}\right)$ is empty. This means that the identity $\mathbf{u}_{\mathbf{k}} \approx \mathbf{v}_{\mathbf{k}}$ has Property $\mathcal{Q}$. The sequence $\mathbf{u} \approx \mathbf{u}_{\mathbf{1}} \approx \mathbf{u}_{\mathbf{2}} \approx \cdots \approx$ $\mathbf{u}_{\mathrm{k}-1} \approx \mathbf{u}_{\mathrm{k}} \approx \mathbf{v}_{\mathrm{k}} \approx \mathbf{v}_{\mathrm{k}-\mathbf{1}} \approx \cdots \approx \mathbf{v}_{\mathbf{2}} \approx \mathbf{v}_{\mathbf{1}} \approx \mathbf{v}$ gives us a derivation of $\mathbf{u} \approx \mathbf{v}$ from $\Sigma$ and from the $\mathcal{Q}$-identity $\mathbf{u}_{\mathbf{k}} \approx \mathbf{v}_{\mathbf{k}}$.

If some variable $x$ occurs $n \geq 0$ times in a word $\mathbf{u}$ then we write $\operatorname{occ}_{\mathbf{u}}(x)=n$ and say that $x$ is $n$-occurring in $\mathbf{u}$. We use letter $t$ with or without subscripts to denote linear (1-occurring) variables. If we use letter $t$ several times in a word, we assume that different occurrences of $t$ represent distinct linear variables.

For each $n>0$ we define $\operatorname{Cont}_{n}(\mathbf{u})=\left\{x \in \mathfrak{A} \mid 0<\operatorname{occ}_{\mathbf{u}}(x) \leq n\right\}$. We use $\operatorname{Lin}(\mathbf{u})$ to denote the set $\operatorname{Cont}_{1}(\mathbf{u})$ of all linear variables in $\mathbf{u}$. We use Non( $\left.\mathbf{u}\right)$ to denote the set of all non-linear variables in $\mathbf{u}$. Evidently, $\operatorname{Cont}(\mathbf{u})=\operatorname{Lin}(\mathbf{u}) \cup \operatorname{Non}(\mathbf{u})$.

For each $n>0$ an identity $\mathbf{u} \approx \mathbf{v}$ is called a $\mathcal{P}_{n}$-identity if it is regular and $\mathbf{u}\left(\operatorname{Cont}_{n}(\mathbf{u})\right)=\mathbf{v}\left(\operatorname{Cont}_{n}(\mathbf{u})\right)$. In particular, an identity $\mathbf{u} \approx \mathbf{v}$ is a $\mathcal{P}_{1}$-identity if and only if $\operatorname{Lin}(\mathbf{u})=\operatorname{Lin}(\mathbf{v}), \operatorname{Non}(\mathbf{u})=\operatorname{Non}(\mathbf{u})$ and the order of linear letters is the same in $\mathbf{u}$ and $\mathbf{v}$. An identity $\mathbf{u} \approx \mathbf{v}$ is called block-balanced if for each variable $x \in \mathfrak{A}$, we have $\mathbf{u}(x, \operatorname{Lin}(\mathbf{u}))=\mathbf{v}(x, \operatorname{Lin}(\mathbf{u}))$.

A block of a word $\mathbf{u}$ is a maximal subword of $\mathbf{u}$ that does not contain any linear letters of $\mathbf{u}$. Evidently, an identity $\mathbf{u} \approx \mathbf{v}$ is block-balanced if and only if it is a balanced $\mathcal{P}_{1}$-identity and each block in $\mathbf{u}$ is a permutation of the corresponding block in $\mathbf{v}$. For each $\mathcal{P}_{1}$-identity $\mathbf{u} \approx \mathbf{v}$ we define

- $\operatorname{Dist}\left(\mathcal{P}_{1} \rightarrow\right.$ block-balanced $)(\mathbf{u} \approx \mathbf{v}):=\{x \in \operatorname{Cont}(\mathbf{u}) \mid \mathbf{u}(x, \operatorname{Lin}(\mathbf{u})) \neq$ $\mathbf{v}(x, \operatorname{Lin}(\mathbf{u}))\}$.

It is easy to see that the set $\operatorname{Dist}\left(\mathcal{P}_{1} \rightarrow\right.$ block-balanced $)(\mathbf{u} \approx \mathbf{v})$ is empty if and only if $\mathbf{u} \approx \mathbf{v}$ is a block-balanced identity.

If $x$ is a non-linear variable in a word $\mathbf{u}$ then we say that $\mathbf{u}$ is $x$-compact if all occurrences of $x$ are collected together in each block of $\mathbf{u}$. For example, the word xxytyxy is $x$-compact while the word $x y y x$ is not. Now we illustrate how to use Lemma 3.1.

Lemma 3.2. If a monoid $S$ satisfies the identities $\left\{\sigma_{1}, \sigma_{\mu}\right\}$ then every identity of $S$ can be derived from some almost-linear identities and block-balanced identities of $S$. 
Proof. Let $\Sigma$ denote the set of all almost-linear identities of $S$ together with $\left\{\sigma_{1}, \sigma_{\mu}\right\}^{\delta}$. Let $\mathbf{u} \approx \mathbf{v}$ be a $\mathcal{P}_{1}$-identity of $S$ which is not block-balanced.

Since $\mathbf{u} \approx \mathbf{v}$ is a $\mathcal{P}_{1}$-identity, we have $\operatorname{Lin}(\mathbf{u})=\operatorname{Lin}(\mathbf{v})$ and $\operatorname{Non}(\mathbf{u})=\operatorname{Non}(\mathbf{v})$. Since $\mathbf{u} \approx \mathbf{v}$ is not block-balanced, for some variable $x$ such that $\operatorname{occ}_{\mathbf{u}}(x)>1$ the identity $\mathbf{u}(x, \operatorname{Lin}(\mathbf{u})) \approx \mathbf{v}(x, \operatorname{Lin}(\mathbf{u}))$ is non-trivial. We may assume that $\mathbf{u}$ is $x$ compact. (Otherwise, by using the identities in $\left\{\sigma_{1}, \sigma_{\mu}\right\}^{\delta}$ and swapping the adjacent occurrences of variables, we move some non-last occurrences of $x$ to the right until we obtain an $x$-compact word).

Since $\mathbf{u}$ is $x$-compact, the word $\mathbf{u}(x, \operatorname{Lin}(\mathbf{u}))$ is applicable to $\mathbf{u}$. So, for some word $\mathbf{u}_{\mathbf{1}}$ we have $\mathbf{u}(x, \operatorname{Lin}(\mathbf{u})) \approx \mathbf{v}(x, \operatorname{Lin}(\mathbf{u})) \vdash \mathbf{u} \approx \mathbf{u}_{\mathbf{1}}$. Notice that $\mathbf{u}_{\mathbf{1}}(x, \operatorname{Lin}(\mathbf{u}))=$ $\mathbf{v}(x, \operatorname{Lin}(\mathbf{u}))$. This means that $\mid \operatorname{Dist}\left(\mathcal{P}_{1} \rightarrow\right.$ block-balanced $)\left(\mathbf{u}_{\mathbf{1}} \approx \mathbf{v}\right)|<| \operatorname{Dist}\left(\mathcal{P}_{1} \rightarrow\right.$ block-balanced $)(\mathbf{u} \approx \mathbf{v}) \mid$.

Lemma 3.1 implies that every $\mathcal{P}_{1}$-identity of $S$ can be derived from $\Sigma$ and from some block-balanced identity of $S$. Since both $\sigma_{1}$ and $\sigma_{\mu}$ are block-balanced identities, every $\mathcal{P}_{1}$-identity of $S$ can be derived from some almost-linear and blockbalanced identities of $S$.

If the word $x y$ is an isoterm for $S$, then every identity of $S$ satisfies property $\mathcal{P}_{1}$. If the word $x y$ is not an isoterm for $S$, then in view of Fact 2.1, we may assume that $S \models x \approx x^{n}$ for some $n>1$ and satisfies only regular identities. Then by using the identity $x \approx x^{n}$, one can transform every word $\mathbf{p}$ into a word $\mathbf{u}$ so that each variable is non-linear in $\mathbf{u}$. This means that every identity of $S$ can be derived from $x \approx x^{n}$ and from a $\mathcal{P}_{1}$-identity of $S$. Consequently, every identity of $S$ can be derived from some almost-linear identities and block-balanced identities of $S$.

For each balanced identity $\mathbf{u} \approx \mathbf{v}$ we define

- Dist(balanced $\rightarrow$ trivial $)(\mathbf{u} \approx \mathbf{v}):=\left\{\{c, d\} \mid c, d \in \operatorname{OccSet}(\mathbf{u}), c<_{\mathbf{u}} d, d<_{\mathbf{v}} c\right\}$. In other words, Dist(balanced $\rightarrow$ trivial $)(\mathbf{u} \approx \mathbf{v})$ is the set of all unstable pairs in a balanced identity $\mathbf{u} \approx \mathbf{v}$. It is easy to see that the set Dist(balanced $\rightarrow$ trivial $)(\mathbf{u} \approx \mathbf{v})$ is empty if and only if $\mathbf{u} \approx \mathbf{v}$ is a trivial identity. To be consistent with [7, 12] we will write $\operatorname{Chaos}(\mathbf{u} \approx \mathbf{v})$ instead of $\operatorname{Dist}($ balanced $\rightarrow \operatorname{trivial})(\mathbf{u} \approx \mathbf{v})$. The following theorem illustrates how to use this function. It can also be easily deduced from Proposition 4.1 in [9].

Theorem 3.3. Every monoid $S$ which satisfies $\left\{\sigma_{1}, \sigma_{\mu}, \sigma_{2}\right\}$ is finitely based by $\left\{\sigma_{1}, \sigma_{\mu}, \sigma_{2}\right\}^{\delta}$ and some almost-linear identities.

Proof. The following statement is reversed in Proposition 6.1.

Claim 1. Every block-balanced identity can be derived from $\left\{\sigma_{1}, \sigma_{\mu}, \sigma_{2}\right\}^{\delta}$.

Proof. Let $\mathbf{u} \approx \mathbf{v}$ be a non-trivial block-balanced identity. Since $\mathbf{u} \approx \mathbf{v}$ is nontrivial, it contains an unstable pair $\{c, d\} \subseteq \operatorname{OccSet}(\mathbf{u})$. In view of Lemma 2.4, we may assume that $c \ll_{\mathbf{u}} d$. Since the identity $\mathbf{u} \approx \mathbf{v}$ is block-balanced, both $c$ and $d$ are occurrences of some non-linear variables $x \neq y$. We swap $c$ and $d$ in $\mathbf{u}$ by using an identity in $\left\{\sigma_{1}, \sigma_{\mu}, \sigma_{2}\right\}^{\delta}$ and obtain a word $\mathbf{u}_{\mathbf{1}}$. Evidently, $\mid \operatorname{Chaos}\left(\mathbf{u}_{\mathbf{1}} \approx\right.$ 
$\mathbf{v})|<|$ Chaos $(\mathbf{u} \approx \mathbf{v}) \mid$. Lemma 3.1 implies that every block-balanced identity can be derived from $\left\{\sigma_{1}, \sigma_{\mu}, \sigma_{2}\right\}^{\delta}$.

Lemma 3.2. Claim 1 and the result of Volkov (Lemma 2.2) immediately imply that $S$ is finitely based by $\left\{\sigma_{1}, \sigma_{\mu}, \sigma_{2}\right\}^{\delta}$ and some almost-linear identities.

Now we use Lemma 3.1 to obtain an accelerated tool for proving that a semigroup is finitely based.

An assignment of Types from 1 to $n$ is a collection of functions $\left\{T_{\mathbf{u}} \mid \mathbf{u} \in\right.$ $\left.\mathfrak{A}^{+}\right\}$such that for each word $\mathbf{u}, T_{\mathbf{u}}$ assigns values in $\{1,2, \ldots, n\}$ to some pairs of occurrences of distinct variables in $\mathbf{u}$.

If $\mathbf{u} \approx \mathbf{v}$ is a balanced identity then $l_{\mathbf{u}, \mathbf{v}}$ is a bijection from $\operatorname{OccSet}(\mathbf{u})$ to $\operatorname{OccSet}(\mathbf{v})$ defined by $l_{\mathbf{u}, \mathbf{v}}\left({ }_{i \mathbf{u}} x\right)={ }_{i \mathbf{v}} x$. We say that a property $\mathcal{P}$ of identities is transitive if an identity $\mathbf{u} \approx \mathbf{v}$ satisfies $\mathcal{P}$ whenever both $\mathbf{u} \approx \mathbf{w}$ and $\mathbf{w} \approx \mathbf{v}$ satisfy $\mathcal{P}$. Let $\mathcal{P}$ be a transitive property of identities which is at least as strong as the property of being a balanced identity. We say that an assignment of Types is $\mathcal{P}$-compatible if it satisfies the following:

(i) if for some $c \neq d \in \operatorname{OccSet}(\mathbf{u})$ the pair $\{c, d\}$ is unstable in some $\mathcal{P}$-identity $\mathbf{u} \approx \mathbf{v}$, then $T_{\mathbf{u}}(\{c, d\})$ is defined;

(ii) for each $\mathcal{P}$-identity $\mathbf{u} \approx \mathbf{v}$ and for each $c \neq d \in \operatorname{OccSet}(\mathbf{u})$ we have $T_{\mathbf{u}}(\{c, d\})=T_{\mathbf{v}}\left(\left\{l_{\mathbf{u}, \mathbf{v}}(c), l_{\mathbf{u}, \mathbf{v}}(d)\right\}\right)$.

Each $\mathcal{P}$-compatible assignment of Types induces a function on $\mathcal{P}$-identities. We say that a $\mathcal{P}$-identity $\mathbf{u} \approx \mathbf{v}$ is of Type $k$ if $k$ is the maximal number such that the identity $\mathbf{u} \approx \mathbf{v}$ contains an unstable pair of Type $k$. If $\mathbf{u} \approx \mathbf{v}$ does not contain any unstable pairs (i.e. trivial) then we say that $\mathbf{u} \approx \mathbf{v}$ is of Type 0 .

Lemma 3.4. Let $\mathcal{P}$ be a transitive property of identities which is at least as strong as the property of being a balanced identity and $\Delta$ be a set of identities. Suppose that one can find a $\mathcal{P}$-compatible assignment of Types from 1 to $n$ so that for each $1 \leq i \leq n$, if a $\mathcal{P}$-identity $\mathbf{u} \approx \mathbf{v}$ contains a critical pair $\{c, d\} \subseteq \operatorname{OccSet}(\mathbf{u})$ of Type $i$ then one can find a word $\mathbf{w}$ such that

(i) $\Delta \vdash \mathbf{u} \approx \mathbf{w}$;

(ii) $\mathbf{w} \approx \mathbf{v}$ is a $\mathcal{P}$-identity;

(iii) the pair $\{c, d\}$ is stable in $\mathbf{w} \approx \mathbf{v}$;

(iv) each pair of Type $\geq i$ is stable in $\mathbf{w} \approx \mathbf{v}$ whenever it is stable in $\mathbf{u} \approx \mathbf{v}$.

Then every $\mathcal{P}$-identity can be derived from $\Delta$.

Proof. For each $1 \leq i \leq n$, we use $\operatorname{Chaos}_{i}(\mathbf{x} \approx \mathbf{y})$ to denote the set of all unstable pairs of Type $i$ in a $\mathcal{P}$-identity $\mathbf{x} \approx \mathbf{y}$.

Claim 2. Let $\mathbf{u} \approx \mathbf{v}$ be a $\mathcal{P}$-identity of Type $k$ for some $1 \leq k \leq n$. Then one can find a word $\mathbf{u}_{\mathbf{1}}$ such that $\Delta \vdash \mathbf{u} \approx \mathbf{u}_{\mathbf{1}}$, the identity $\mathbf{u}_{\mathbf{1}} \approx \mathbf{v}$ is a $\mathcal{P}$-identity of Type at most $k$ and Chaos $_{k}\left(\mathbf{u}_{\mathbf{1}} \approx \mathbf{v}\right)$ is a proper subset of Chaos $(\mathbf{u} \approx \mathbf{v})$.

Proof. Since $\mathbf{u} \approx \mathbf{v}$ is of Type $k>0$, it contains an unstable pair of Type $k$. Then by Lemma 2.4, the identity $\mathbf{u} \approx \mathbf{v}$ contains a critical pair $\left\{a_{1}, b_{1}\right\} \subseteq \operatorname{OccSet}(\mathbf{u})$. 
The pair $\left\{a_{1}, b_{1}\right\}$ is of Type $T_{1} \in\{1,2, \ldots, k\}$. By our assumption, one can find a word $\mathbf{p}_{\mathbf{1}}$ such that $\Delta \vdash \mathbf{u} \approx \mathbf{p}_{\mathbf{1}}$, for each $i>T_{1}, \operatorname{Chaos}_{i}\left(\mathbf{p}_{\mathbf{1}} \approx \mathbf{v}\right)$ is a subset of $\operatorname{Chaos}_{i}(\mathbf{u} \approx \mathbf{v})$ and $\operatorname{Chaos}_{T_{1}}\left(\mathbf{p}_{\mathbf{1}} \approx \mathbf{v}\right)$ is a proper subset of $\operatorname{Chaos}_{T_{1}}(\mathbf{u} \approx \mathbf{v})$.

If $\operatorname{Chaos}_{k}\left(\mathbf{p}_{\mathbf{1}} \approx \mathbf{v}\right)$ is a proper subset of $\operatorname{Chaos}_{k}(\mathbf{u} \approx \mathbf{v})$ then we are done. Otherwise, $T_{1}<k, \operatorname{Chaos}_{k}\left(\mathbf{p}_{\mathbf{1}} \approx \mathbf{v}\right)=\operatorname{Chaos}_{k}(\mathbf{u} \approx \mathbf{v})$ and in view of Lemma 2.4, the identity $\mathbf{p}_{\mathbf{1}} \approx \mathbf{v}$ contains a critical pair $\left\{a_{2}, b_{2}\right\} \subseteq \operatorname{OccSet}\left(\mathbf{p}_{\mathbf{1}}\right)$. The pair $\left\{a_{2}, b_{2}\right\}$ is of Type $T_{2} \in\{1,2, \ldots, k\}$. By our assumption, one can find a word $\mathbf{p}_{\mathbf{2}}$ such that $\Delta \vdash \mathbf{p}_{\mathbf{1}} \approx \mathbf{p}_{\mathbf{2}}$, for each $i>T_{2}, \operatorname{Chaos}_{i}\left(\mathbf{p}_{\mathbf{1}} \approx \mathbf{v}\right)$ is a subset of $\operatorname{Chaos}_{i}\left(\mathbf{p}_{\mathbf{2}} \approx \mathbf{v}\right)$ and $\operatorname{Chaos}_{T_{2}}\left(\mathbf{p}_{\mathbf{2}} \approx \mathbf{v}\right)$ is a proper subset of $\operatorname{Chaos}_{T_{2}}\left(\mathbf{p}_{\mathbf{1}} \approx \mathbf{v}\right)$. And so on.

If for some $j>0, \operatorname{Chaos}_{k}\left(\mathbf{p}_{\mathbf{j}} \approx \mathbf{v}\right)$ is a proper subset of $\operatorname{Chaos}_{k}(\mathbf{u} \approx \mathbf{v})$ then we are done. Otherwise, we obtain an infinite sequence of words $\mathbf{p}_{\mathbf{1}}, \mathbf{p}_{\mathbf{2}}, \ldots$ and numbers $T_{1}, T_{2}, \ldots$ such that for each $j>0$ we have $\operatorname{Chaos}_{k}\left(\mathbf{p}_{\mathbf{j}} \approx \mathbf{v}\right)=\operatorname{Chaos}_{k}(\mathbf{u} \approx \mathbf{v})$ and $0<T_{j}<k$.

Let $m<k$ be the biggest number that repeats in this sequence infinite number of times. This means that starting with some number $Q$ big enough, we do not see any critical pairs of Types bigger than $m$ and that one can find a subsequence $Q<$ $j_{1}<j_{2}<\ldots$ so that $m=T_{j_{1}}=T_{j_{2}}=T_{j_{3}}=\ldots$. Then for each $g=1,2, \ldots$, the set $\operatorname{Chaos}_{m}\left(\mathbf{p}_{\mathbf{j g}} \approx \mathbf{v}\right)$ is a proper subset of $\operatorname{Chaos}_{m}\left(\mathbf{p}_{\mathbf{j}_{\mathbf{g}-1}} \approx \mathbf{v}\right)$. This means that the number of critical pairs of Type $m$ must be decreasing to zero. A contradiction.

In view of Lemma 3.1, for each $1 \leq k \leq n$, every $\mathcal{P}$-identity of Type $k$ can be derived from $\Delta$ and from a $\mathcal{P}$-identity of Type at most $(k-1)$. By induction, every $\mathcal{P}$-identity can be derived from $\Delta$.

If $x$ and $y$ are non-linear variables in a word $\mathbf{u}$ then we say that $\mathbf{u}$ is $x y$-compact if all occurrences of $x$ and $y$ are collected together in each block of $\mathbf{u}$. For example, the word pxxyztpyxyz is $x y$-compact while the word $x y z y x z$ is not.

Theorem 3.5. [9, Theorem 1.1] Every monoid $S$ which satisfies $\left\{\sigma_{1}, \sigma_{\mu}\right\}$ (or dually, $\left.\left\{\sigma_{\mu}, \sigma_{2}\right\}\right)$ is finitely based by some almost-linear identities and by some block-balanced identities with two non-linear variables.

Proof. By Lemma 3.2, every identity of $S$ can be derived from some almost-linear identities of $S$ and from some block-balanced identities of $S$. By the result of Volkov (Lemma 2.2) all almost-linear identities of $S$ can be derived from a finite set of almost-linear identities of $S$.

Claim 3. All block-balanced identities of $S$ can be derived from its block-balanced identities with two non-linear variables.

Proof. We assign a Type to each pair $\{c, d\} \subseteq \operatorname{OccSet}(\mathbf{u})$ of occurrences of distinct non-linear variables $x \neq y$ in a word $\mathbf{u}$ as follows: $\{c, d\}$ is of Type 2 if $\{c, d\}=$ $\{$ lastu $x$, lastu $y\}$ and of Type 1 otherwise. It is easy to see that this assignment of Types is compatible with the property of being a block-balanced identity.

Let $\Delta$ be the set of all block-balanced identities of $S$ with two non-linear variables. Let $\mathbf{u} \approx \mathbf{v}$ be a block-balanced identity of $S$ and $\{c, d\} \subseteq \operatorname{OccSet}(\mathbf{u})$ be a 
critical pair in $\mathbf{u} \approx \mathbf{v}$. If $\{c, d\}$ is of Type 1 , then by using an identity from $\left\{\sigma_{1}, \sigma_{\mu}\right\}^{\delta}$ we swap $c$ and $d$ in $\mathbf{u}$ and obtain a word $\mathbf{w}$. Evidently, the word $\mathbf{w}$ satisfies all three requirements of Lemma 3.4.

If $\{c, d\}$ is of Type 2 , then $c={ }_{\text {last }} x$ and $d={ }_{\text {last }} y$. We may assume that $\mathbf{u}$ is $x y$ compact. (Otherwise, by using the identities in $\left\{\sigma_{1}, \sigma_{\mu}\right\}^{\delta}$ and swapping the adjacent occurrences of variables, we move some non-last occurrences of $x$ and $y$ to the right until we obtain an $x y$-compact word.) If we apply the identity $\mathbf{u}(x, y, \operatorname{Lin}(\mathbf{u})) \approx$ $\mathbf{v}(x, y, \operatorname{Lin}(\mathbf{u}))$ to $\mathbf{u}$ we obtain a word $\mathbf{w}$ which satisfies all three requirements of Lemma 3.4.

Lemma 3.4 implies that all block-balanced identities of $S$ can be derived from its block-balanced identities with two non-linear variables.

If $\mathbf{u}$ is a word with two non-linear variables then by using the identities $\left\{\sigma_{1}, \sigma_{\mu}\right\}^{\delta}$ and commuting adjacent occurrences of variables, the word $\mathbf{u}$ can be transform into one side of an identity of the form (11). By the result of Lee (Lemma 2.3), all identities of $S$ of the form (11) can be derived from a finite subset. Therefore, the monoid $S$ is finitely based by some almost-linear identities and by some block-balanced identities with two non-linear variables.

\section{Finitely based subvarieties of $\operatorname{var}\left\{\sigma_{1}, \sigma_{2}\right\}$}

We say that an identity $\mathbf{u} \approx \mathbf{v}$ has Property $\mathcal{P}_{1,2}$ if $\operatorname{Lin}(\mathbf{u})=\operatorname{Lin}(\mathbf{v}), \operatorname{Non}(\mathbf{u})=$ $\operatorname{Non}(\mathbf{v})$ and for each $x, y \in \operatorname{Cont}(\mathbf{u})$ we have $\left({ }_{1 \mathbf{u}} x\right)<_{\mathbf{u}}\left({ }_{\text {last }} y\right)$ iff $\left({ }_{1 \mathbf{v}} x\right)<_{\mathbf{v}}\left({ }_{\text {last }} y\right)$. Evidently, Property $\mathcal{P}_{1,2}$ is stronger than $\mathcal{P}_{1}$. The following lemma will be reversed in Proposition 6.2.

Lemma 4.1. Every block-balanced $\mathcal{P}_{1,2}$-identity can be derived from $\left\{\sigma_{1}, \sigma_{2}\right\}^{\delta}$.

Proof. Let $\mathbf{u} \approx \mathbf{v}$ be a non-trivial block-balanced $\mathcal{P}_{1,2}$-identity. Since $\mathbf{u} \approx \mathbf{v}$ is non-trivial, it contains an unstable pair $\{c, d\} \subseteq \operatorname{OccSet}(\mathbf{u})$. In view of Lemma 2.4. we may assume that $c \ll \mathbf{u} d$. Since $\mathbf{u} \approx \mathbf{v}$ is block-balanced, both $c$ and $d$ are occurrences of some non-linear variables $x \neq y \in \operatorname{Non}(\mathbf{u})$. Since $\mathbf{u} \approx \mathbf{v}$ has Property $\mathcal{P}_{1,2}$, the pair $\{c, d\}$ is not of the form $\left\{{ }_{1 \mathbf{u}} x\right.$, last $\left.\mathbf{u} y\right\}$. Therefore, one can swap $c$ and $d$ in $\mathbf{u}$ by using an identity in $\left\{\sigma_{1}, \sigma_{2}\right\}^{\delta}$ and obtain a word $\mathbf{u}_{\mathbf{1}}$. Notice that $\operatorname{Chaos}\left(\mathbf{u}_{\mathbf{1}} \approx \mathbf{w}\right)$ is a proper subset of Chaos $(\mathbf{u} \approx \mathbf{v})$. By Lemma 3.1, every block-balanced $\mathcal{P}_{1,2}$-identity can be derived from $\left\{\sigma_{1}, \sigma_{2}\right\}^{\delta}$.

For $n>0$, a word $\mathbf{u}$ is called $n$-limited if each variable occurs in $\mathbf{u}$ at most $n$ times. An identity is called $n$-limited if both sides of this identity are $n$-limited words. We use $A_{0}^{1}$ to denote the monoid obtained by adjoining an identity element to the semigroup $A_{0}=\langle a, b \mid a a=a, b b=b, a b=0\rangle$ of order four.

Proposition 4.2. For a monoid $S$ the following are equivalent:

(i) $E q(S)=J_{2}$;

(ii) $\operatorname{Eq}(S)$ is the set of all $\mathcal{P}_{1,2}$-identities; 
(iii) $S$ is finitely based by $\left\{\sigma_{1}, \sigma_{2}, x t_{1} x t_{2} x \approx x t_{1} t_{2} x\right\}^{\delta}$;

(iv) $S$ is equationally equivalent to $A_{0}^{1}$.

Proof. (i) $\rightarrow$ (ii) Take $(\mathbf{u} \approx \mathbf{v}) \in J_{2}$. Since the word $t$ is an isoterm for $J_{2}$, we have that $\operatorname{Lin}(\mathbf{u})=\operatorname{Lin}(\mathbf{v})$ and $\operatorname{Non}(\mathbf{u})=\operatorname{Non}(\mathbf{v})$.

Suppose that for some $x \neq y \in \operatorname{Cont}(\mathbf{u})$ we have $\left({ }_{\text {last } \mathbf{u}} x\right)<_{\mathbf{u}}\left({ }_{1 \mathbf{u}} y\right)$ but $\left({ }_{1 \mathbf{v}} y\right)<_{\mathbf{v}}$ $\left(\operatorname{last}_{\mathbf{v}} x\right)$. Then the word $\mathbf{u}$ deletes to $x^{n} y^{m}$ for some $n, m>0$. Notice that the word $\mathbf{u}$ does not contain the scattered subword $y x$. On the other hand, the word $\mathbf{v}$ contains the scattered subword $y x$. To avoid a contradiction we conclude that $\mathbf{u} \approx \mathbf{v}$ is a $\mathcal{P}_{1,2}$-identity.

Conversely, let $\mathbf{u} \approx \mathbf{v}$ be a $\mathcal{P}_{1,2}$-identity. If $\mathbf{u}$ contains a scattered subword $x y$ for some $x, y \in \operatorname{Cont}(\mathbf{u})$ then $\left({ }_{1} \mathbf{u} x\right)<_{\mathbf{u}}$ (lastu$\left.y\right)$. Since $\mathbf{u} \approx \mathbf{v}$ is a $\mathcal{P}_{1,2}$-identity, we have $\left({ }_{1 \mathbf{v}} x\right)<_{\mathbf{v}}\left({ }_{\text {last } \mathbf{v}} y\right)$. Consequently, $x y$ is a scattered subword of $\mathbf{v}$.

(ii) $\rightarrow$ (iii) It is easy to see that every $\mathcal{P}_{1}$-identity is a consequence of $\left\{x t_{1} x t_{2} x \approx\right.$ $\left.x t_{1} t_{2} x\right\}^{\delta}$ and a 2-limited balanced identity. It is also easy to see that a 2 -limited balanced $\mathcal{P}_{1,2}$-identity is block-balanced. Therefore, every $\mathcal{P}_{1,2}$-identity is a consequence of $\left\{x t_{1} x t_{2} x \approx x t_{1} t_{2} x\right\}^{\delta}$ and of a 2 -limited block-balanced $\mathcal{P}_{1,2}$-identity. So, by Lemma 4.1, every identity of $S$ is a consequence of $\left\{x t_{1} x t_{2} x \approx x t_{1} t_{2} x, \sigma_{1}, \sigma_{2}\right\}^{\delta}$.

$\left(\right.$ iii) $\rightarrow$ (iv) According to Proposition 3.2(a) in [4] the set $\left\{x t_{1} x t_{2} x \approx x t_{1} t_{2} x, \sigma_{1}, \sigma_{2}\right\}^{\delta}$ is a finite basis for the monoid $A_{0}^{1}$.

(iv) $\rightarrow$ (i) According to Theorem 3.5 in [2] the set $J_{2}$ is finitely based by $\left\{x t_{1} x t_{2} x \approx x t_{1} t_{2} x,(x y)^{2} \approx(y x)^{2}\right\}$. It is easy to see that this set of identities is equivalent to $\left\{x t_{1} x t_{2} x \approx x t_{1} t_{2} x, \sigma_{1}, \sigma_{2}\right\}^{\delta}$.

We say that an identity $\mathbf{u} \approx \mathbf{v}$ has property $\mathcal{P}_{1 b}$ if $\operatorname{Lin}(\mathbf{u})=\operatorname{Lin}(\mathbf{v}), \operatorname{Non}(\mathbf{u})=$ $\operatorname{Non}(\mathbf{v}), \mathbf{u}(\operatorname{Lin}(\mathbf{u}))=\mathbf{v}(\operatorname{Lin}(\mathbf{u}))$ and if for some variable $x \in \operatorname{Non}(\mathbf{u})$ the identity $\mathbf{u}(x, \operatorname{Lin}(\mathbf{u})) \approx \mathbf{v}(x, \operatorname{Lin}(\mathbf{u}))$ is non-trivial then all occurrences of $x$ in $\mathbf{u}$ (in $\mathbf{v}$ ) belong to the same block of $\mathbf{u}$ ( of $\mathbf{v}$ ). Evidently, Property $\mathcal{P}_{1 b}$ is stronger than $\mathcal{P}_{1}$ but weaker than the property of being a block-balanced identity.

Lemma 4.3. Let $S$ be a monoid such that $S \models\left\{\sigma_{1}, \sigma_{2}\right\}$. Then every $\mathcal{P}_{1}$-identity of $S$ can be derived from some almost-linear identities of $S$, from $\left\{\sigma_{1}, \sigma_{2}\right\}^{\delta}$ and from a $\mathcal{P}_{1 b}$-identity of $S$.

Proof. Let $\Sigma$ denote the set of all almost-linear identities of $S$ together with $\left\{\sigma_{1}, \sigma_{2}\right\}^{\delta}$.

Let $\mathbf{u} \approx \mathbf{v}$ be a $\mathcal{P}_{1}$-identity of $S$ which does not have Property $\mathcal{P}_{1 b}$. This means that $\operatorname{Lin}(\mathbf{u})=\operatorname{Lin}(\mathbf{v}), \operatorname{Non}(\mathbf{u})=\operatorname{Non}(\mathbf{v}), \mathbf{u}(\operatorname{Lin}(\mathbf{u}))=\mathbf{v}(\operatorname{Lin}(\mathbf{u}))$, for some variable $x \in \operatorname{Non}(\mathbf{u})$ the identity $\mathbf{u}(x, \operatorname{Lin}(\mathbf{u})) \approx \mathbf{v}(x, \operatorname{Lin}(\mathbf{u}))$ is non-trivial and either ${ }_{1 \mathbf{u}} x$ and $\operatorname{last}_{\mathbf{u}} x$ belong to different blocks of $\mathbf{u}$ or ${ }_{1 \mathbf{v}} x$ and ${ }_{\text {last } \mathbf{v}} x$ belong to different blocks of $\mathbf{v}$.

By symmetry, we may assume that ${ }_{1 \mathbf{u}} x$ and lastu $x$ belong to different blocks of $\mathbf{u}$. We may also assume that $\mathbf{u}$ is $x x$-compact. (Otherwise, we fix the first and the last occurrences of $x$ and by using the identities in $\left\{\sigma_{1}, \sigma_{2}\right\}^{\delta}$ we move some non-first and non-last occurrences of $x$ until we obtain an $x x$-compact word).

The word $\mathbf{u}(x, \operatorname{Lin}(\mathbf{u}))$ is applicable to $\mathbf{u}$. So, for some word $\mathbf{u}_{\mathbf{1}}$ we have $\mathbf{u}(x, \operatorname{Lin}(\mathbf{u})) \approx$ $\mathbf{v}(x, \operatorname{Lin}(\mathbf{u})) \vdash \mathbf{u} \approx \mathbf{u}_{\mathbf{1}}$. Notice that $\mathbf{u}_{\mathbf{1}}(x, \operatorname{Lin}(\mathbf{u}))=\mathbf{v}(x, \operatorname{Lin}(\mathbf{u}))$. This means that 
$\left|\operatorname{Dist}\left(\mathcal{P}_{1} \rightarrow \mathcal{P}_{1 b}\right)\left(\mathbf{u}_{\mathbf{1}} \approx \mathbf{v}\right)\right|<\left|\operatorname{Dist}\left(\mathcal{P}_{1} \rightarrow \mathcal{P}_{1 b}\right)(\mathbf{u} \approx \mathbf{v})\right|$. By Lemma 3.1, every identity of $S$ can be derived from $\Sigma$ and from some $\mathcal{P}_{1 b}$-identity of $S$.

Theorem 4.4. Let $S$ be a monoid such that $S \models\left\{\sigma_{1}, \sigma_{2}\right\}$. Suppose also that for some $k \geq 0$ the word $x^{k} y^{k}$ is an isoterm for $S$ and $S \models\left\{t_{1} x t_{2} x \ldots t_{k+1} x \approx\right.$ $\left.x^{k+1} t_{1} t_{2} \ldots t_{k+1}, x^{k+1} \approx x^{k+2}\right\}$.

Then $S$ is finitely based by some almost-linear identities together with $\left\{\sigma_{1}, \sigma_{2}\right\}^{\delta}$.

Proof. It is easy to see that every identity of $S$ can be derived from $\left\{t_{1} x t_{2} x \ldots t_{k+1} x \approx\right.$ $\left.x^{k+1} t_{1} t_{2} \ldots t_{k+1}, x^{k+1} \approx x^{k+2}\right\}^{\delta}$ and a $k$-limited identity of $S$. Since the word $x^{k} y^{k}$ is an isoterm for $S$, every $k$-limited identity of $S$ has property $\mathcal{P}_{1,2}$. Consequently, it has property $\mathcal{P}_{1}$. Since the words $x^{k} t$ and $t x^{k}$ are isoterms for $S$, every $k$-limited $\mathcal{P}_{1, b}$-identity of $S$ is block-balanced. By Lemma 4.3, every $k$-limited identity of $S$ can be derived from some almost-linear identities of $S$, from $\left\{\sigma_{1}, \sigma_{2}\right\}^{\delta}$ and from some block-balanced identity of $S$. Now Lemma 4.1 and the result of Volkov (Lemma 2.2) imply that $S$ is finitely based by some almost-linear identities together with $\left\{\sigma_{1}, \sigma_{2}\right\}^{\delta}$.

Theorem 4.5. Let $\mathcal{V}$ be a monoid subvariety of $\operatorname{var}\left\{\sigma_{1}, \sigma_{2}\right\}$ which contains the monoid $A_{0}^{1}$. Then $\mathcal{V}$ is finitely based by some almost-linear identities together with $\left\{\sigma_{1}, \sigma_{2}\right\}^{\delta}$ in each of the following cases:

(i) $\mathcal{V}$ is non-periodic;

(ii) $\mathcal{V}$ is aperiodic and for some $0<d<m$ and $c+p>m, \mathcal{V} \models x^{m-d} t x^{d} \approx x^{c} t x^{p}$ where $m>1$ is the minimal such that $\mathcal{V} \models x^{m} \approx x^{m+1}$.

Proof. Proposition 4.2 implies that every identity of $\mathcal{V}$ is a $\mathcal{P}_{1,2}$-identity and consequently, is a $\mathcal{P}_{1}$-identity. By Lemma 4.3, every identity of $\mathcal{V}$ can be derived from some almost-linear identities of $\mathcal{V}$, from $\left\{\sigma_{1}, \sigma_{2}\right\}^{\delta}$ and from some $\mathcal{P}_{1 b}$-identity of $\mathcal{V}$.

If $\mathcal{V}$ is non-periodic, then every identity of $\mathcal{V}$ is balanced. Now suppose that $\mathcal{V}$ is aperiodic. Let $\mathbf{u} \approx \mathbf{v}$ be a $\mathcal{P}_{1 b}$-identity of $\mathcal{V}$.

Claim 4. The identity $\mathbf{u} \approx \mathbf{v}$ can be derived from $\left\{\sigma_{1}, \sigma_{2}\right\}^{\delta}$ and from a balanced $\mathcal{P}_{1 b}$-identity of $S$.

Proof. If the identity $\mathbf{u} \approx \mathbf{v}$ is not balanced, then for some variable $x$ we have $\mathbf{u}(x) \neq \mathbf{v}(x)$. Since $\mathbf{u} \approx \mathbf{v}$ is a $\mathcal{P}_{1 b}$-identity, all occurrences of $x$ in $\mathbf{u}$ belong to the same block of $\mathbf{u}$ and all occurrences of $x$ in $\mathbf{v}$ belong to the same block of $\mathbf{v}$. By the minimality of $m$ we have $\operatorname{occ}_{\mathbf{u}}(x) \geq m$ and $\operatorname{occ}_{\mathbf{v}}(x) \geq m$.

We may assume that the variable $x$ occurs at most $m+1$ times in $\mathbf{u}$ and in $\mathbf{v}$. (If occ $_{\mathbf{u}}(x)=k>m+1$ then by using $\left\{\sigma_{1}, \sigma_{2}\right\}^{\delta}$ and moving non-last and non-first occurrences of $x$ to the left one can collect the first $k-1$ occurrences of $x$ together and apply $\left.x^{k-1} \approx x^{m}\right)$. Since the identity $\mathbf{u}(x) \approx \mathbf{v}(x)$ is non-trivial, we may assume that $\operatorname{occ}_{\mathbf{u}}(x)=m$ and $\operatorname{occ}_{\mathbf{v}}(x)=m+1$.

By our assumption, we have that $\mathcal{V} \models x^{m-d} t x^{d} \approx x^{c} t x^{p}$ for some $0<d<m$ and $c+p>m$. By using $\left\{\sigma_{1}, \sigma_{2}\right\}^{\delta}$ we collect the first $m-d$ occurrences of $x$ in $\mathbf{u}$ together and the last $d$ occurrences of $x$ together and obtain a word $\mathbf{w}$. Now we 
apply $x^{m-d} t x^{d} \approx x^{c} t x^{p}$ to $\mathbf{w}$ and obtain a word $\mathbf{q}$. Notice that $\operatorname{occ}_{\mathbf{q}}(x)=c+p$. If $c+p>m+1$ then by using $\left\{\sigma_{1}, \sigma_{2}\right\}^{\delta}$ and $x^{c+p-1} \approx x^{m}$ we obtain a word $\mathbf{u}_{\mathbf{1}}$ such that $\operatorname{occ}_{\mathbf{u}_{1}}(x)=m+1$.

Notice that $\mathbf{u}_{\mathbf{1}}(x)=\mathbf{v}(x)$. This means that $\mid \operatorname{Dist}\left(\mathcal{P}_{1 b} \rightarrow\right.$ balanced $)\left(\mathbf{u}_{\mathbf{1}} \approx \mathbf{v}\right) \mid<$ $\mid \operatorname{Dist}\left(\mathcal{P}_{1 b} \rightarrow\right.$ balanced $)(\mathbf{u} \approx \mathbf{v}) \mid$. By Lemma 3.1 , every $\mathcal{P}_{1 b}$-identity of $S$ can be derived from some almost-linear identities of $S$, from $\left\{\sigma_{1}, \sigma_{2}\right\}^{\delta}$ and from some balanced $\mathcal{P}_{1 b}$-identity of $S$.

So, every identity of $\mathcal{V}$ can be derived from some almost-linear identities of $\mathcal{V}$, from $\left\{\sigma_{1}, \sigma_{2}\right\}^{\delta}$ and from some balanced $\mathcal{P}_{1 b}$-identity $\mathbf{p} \approx \mathbf{q}$ of $\mathcal{V}$. Since $\mathbf{p} \approx \mathbf{q}$ has Property $\mathcal{P}_{1,2}$, it is block-balanced. So, every identity of $\mathcal{V}$ can be derived from some almost-linear identities of $\mathcal{V}$, from $\left\{\sigma_{1}, \sigma_{2}\right\}^{\delta}$ and from some block-balanced identity of $\mathcal{V}$.

Now Lemma 4.1 and the result of Volkov (Lemma 2.2) imply that $\mathcal{V}$ is finitely based by some almost-linear identities together with $\left\{\sigma_{1}, \sigma_{2}\right\}^{\delta}$.

Corollary 4.6. Let $S$ be a monoid such that $S \models\left\{\sigma_{1}, \sigma_{2}\right\}$ and for each $k>0$ the word $x^{k} y^{k}$ is an isoterm for $S$. Then $S$ is finitely based by some almost-linear identities together with $\left\{\sigma_{1}, \sigma_{2}\right\}^{\delta}$.

Proof. Since the word $x^{k} y^{k}$ is an isoterm for $S$, each identity of $S$ has Property $\mathcal{P}_{1,2}$. In view of Proposition 4.2, the variety var $S$ contains the monoid $A_{0}^{1}$. Since $S$ is non-periodic, $S$ is finitely based by some almost-linear identities together with $\left\{\sigma_{1}, \sigma_{2}\right\}^{\delta}$ by Theorem 4.5.

The next statement can be easily verified and is generalized in [14, Theorem 7.3].

Fact 4.7. For a set of words $W$ we have $S(W) \models\left\{\sigma_{1}, \sigma_{2}\right\}$ if and only if every adjacent (unordered) pair of occurrences (if any) of two non-linear variables $x \neq y$ in each word in $W$ is of the form $\{1 \mathbf{u} x$, lastu $y\}$.

Theorem 4.8. Let $W$ be a set of words as in Fact 4.7. Let $m>0$ be the maximal integer for which there is $a \in \mathfrak{A}$ such that $a^{m}$ is a subword of a word in $W$.

Then the direct product $S=A_{0}^{1} \times S(W)$ is finitely based if and only if either $m$ is infinite or $m$ is finite and for some $0<d<m+1$ the word $b^{m+1-d} \mathbf{T} b^{d}$ is not a subword of any word in $W$ for any $b \in \mathfrak{A}$ and $\mathbf{T} \in \mathfrak{A}^{+}$.

Proof. If $m$ is infinite then $S$ is finitely based by Theorem 4.5, Assume that $m$ is finite.

If for each $0<d<m$ the word $x^{m+1-d} t x^{d}$ is an isoterm for $S$ then $S$ is non-finitely based by Corollary 7.3 in [13]. If for some $0<d<m+1$ the word $x^{m+1-d} t x^{d}$ is not an isoterm for $S$ then $S \models x^{m+1-d} t x^{d} \approx x^{m+2} t$. Therefore, the aperiodic monoid $S$ is finitely based by Theorem 4.5 .

Theorem 4.8 immediately implies the following. 
Example 4.9. Consider the following sequence of monoids: $M_{1}=A_{0}^{1}, M_{2}=$ $A_{0}^{1} \times S(\{a t a\}), M_{3}=A_{0}^{1} \times S\left(\left\{a^{2} t a\right\}\right), M_{4}=A_{0}^{1} \times S\left(\left\{a^{2} t a, a t a^{2}\right\}\right), M_{5}=A_{0}^{1} \times$ $S\left(\left\{a^{3} t a, a t a^{3}\right\}\right), M_{6}=A_{0}^{1} \times S\left(\left\{a^{3} t a, a t a^{3}, a^{2} t a^{2}\right\}\right), M_{7}=A_{0}^{1} \times S\left(\left\{a^{4} t a, a t a^{4}, a^{3} t a^{2}\right\}\right)$, $M_{8}=A_{0}^{1} \times S\left(\left\{a^{4} t a, a t a^{4}, a^{3} t a^{2}, a^{2} t a^{3}\right\}\right), \ldots$

Then for each $k=1,2, \ldots$, the monoid $M_{k}$ is a submonoid of $M_{k+1}$ and for each $i=0,1,2, \ldots$ the monoid $M_{2 i+1}$ is finitely based while the monoid $M_{2 i}$ is non-finitely based.

We say that a pair of variables $\{x, y\}$ is $b$-unstable in a word $\mathbf{u}$ with respect to a semigroup $S$ if $S$ satisfies a block-balanced identity of the form $\mathbf{u} \approx \mathbf{v}$ such that $\mathbf{u}(x, y) \neq \mathbf{v}(x, y)$.

Theorem 4.10. Let $S$ be a monoid such that $S \models\left\{\sigma_{1}, \sigma_{2}\right\}$ and the word $x y$ is an isoterm for $S$. Suppose that $S$ satisfies the following conditions:

(i) If for some $m, n>1$, the word $x^{m} y^{n}$ is not an isoterm for $S$ then for some $0<d<m$ and $0<c<n$, S satisfies $x^{d} t x^{m-d} y^{n-c} t y^{c} \approx x^{d} t y^{n-c} x^{m-d} t y^{c}$;

(ii) If for some almost-linear word $\mathbf{A} x$ with $\operatorname{occ}_{\mathbf{A}}(x)>0$ the pair $\{x, y\}$ is $b$ unstable in $\mathbf{A} x y^{k}$ with respect to $S$ then for some $0<c<k$, S satisfies $\mathbf{A} x y^{c} t y^{k-c} \approx$ A $y x y^{c-1} t y^{k-c}$;

(iii) If for some almost-linear word $y \mathbf{B}$ with $\operatorname{occ}_{\mathbf{B}}(y)>0$ the pair $\{x, y\}$ is $b$ unstable in $x^{k} y \mathbf{B}$ with respect to $S$ then for some $0<p<k, S$ satisfies $x^{k-p} t x^{p} y \mathbf{B} \approx$ $x^{k-p} t x^{p-1} y x \mathbf{B}$.

Then $S$ is finitely based by some almost-linear identities and by some blockbalanced identities with two non-linear variables.

Proof. Since the word $x y$ is an isoterm for $S$ every identity of $S$ has Property $\mathcal{P}_{1}$.

Claim 5. Every identity of $S$ can be derived from some almost-linear and blockbalanced identities of $S$.

Proof. By Lemma 4.3, every identity of $S$ can be derived from some almost-linear identities of $S$, from $\left\{\sigma_{1}, \sigma_{2}\right\}^{\delta}$ and from a $\mathcal{P}_{1 b^{-}}$identity of $S$. Let $\mathbf{u} \approx \mathbf{v}$ be a $\mathcal{P}_{1 b^{-}}$ identity of $S$. Then $\operatorname{Lin}(\mathbf{u})=\operatorname{Lin}(\mathbf{v}), \operatorname{Non}(\mathbf{u})=\operatorname{Non}(\mathbf{v})$ and $\mathbf{u}(\operatorname{Lin}(\mathbf{u}))=\mathbf{v}(\operatorname{Lin}(\mathbf{u}))$. If the identity $\mathbf{u} \approx \mathbf{v}$ is not block-balanced, for some variable $x$ the identity $\mathbf{u}(x, \operatorname{Lin}(\mathbf{u})) \approx \mathbf{v}(x, \operatorname{Lin}(\mathbf{u}))$ is not trivial. Since $\mathbf{u} \approx \mathbf{v}$ is a $\mathcal{P}_{1 b}$-identity, all occurrences of $x$ in $\mathbf{u}$ are in the same block of $\mathbf{u}$. Therefore, for some $t \in \operatorname{Lin}(\mathbf{u})$ and some $k>1$ the word $\mathbf{u}(x)=x^{k} t$ is not an isoterm for $S$. By Condition (i), for some $0<d<k$ we have $S \models x^{d} t x^{k-d} y t y \approx x^{d} t y x^{k-d} t y$.

We collect all $k$ occurrences of $x$ in $\mathbf{u}$ together as follows. First, by using some identities in $\left\{\sigma_{1}, \sigma_{2}\right\}^{\delta}$ and moving the occurrences of $x$ other than ${ }_{1 \mathbf{u}} x$ and ${ }_{k \mathbf{u}} x$ to the left toward the first occurrence of $x$, we obtain a word $\mathbf{r}^{\prime}$ where the first $d$ occurrences of $x$ are collected together. In a similar way we collect the last $k-d$ occurrences of $x$ together and obtain a word $\mathbf{r}$.

Now we move $x^{k-d}$ to the left by commuting it with adjacent occurrences of variables other than $x$. Suppose that $q \ll_{\mathbf{r}}\left({ }_{(k-d) \mathbf{r}} x\right)$ where $q$ is an occurrence of some variable $z \neq x$. If $q$ is not the first occurrence of $z$ then by using an identity 
in $\left\{\sigma_{2}\right\}^{\delta}$ we obtain a word $\mathbf{p}$ so that $\left({ }_{k \mathbf{p}} x\right) \ll_{\mathbf{p}} q$. If $q$ is the first occurrence of $z$ then by using the identity $x^{d} t x^{k-d} z t z \approx x^{d} t z x^{k-d} t z$ we obtain a word $\mathbf{p}$ such that $\left(k_{\mathbf{p}} x\right) \ll_{\mathbf{p}} q$. And so on, until we obtain a word $\mathbf{w}$ where all $k$ occurrences of $x$ are collected together.

Now we apply the identity $\mathbf{w}(x, \operatorname{Lin}(\mathbf{u})) \approx \mathbf{v}(x, \operatorname{Lin}(\mathbf{u}))$ to $\mathbf{w}$ and obtain a word $\mathbf{u}_{\mathbf{1}}$. Notice that $\mathbf{u}_{\mathbf{1}}(x, \operatorname{Lin}(\mathbf{u}))=\mathbf{v}(x, \operatorname{Lin}(\mathbf{u}))$. This means that $\mid \operatorname{Dist}\left(\mathcal{P}_{1 b} \rightarrow\right.$ blockbalanced $)\left(\mathbf{u}_{\mathbf{1}} \approx \mathbf{v}\right)|<| \operatorname{Dist}\left(\mathcal{P}_{1 b} \rightarrow\right.$ block-balanced $)(\mathbf{u} \approx \mathbf{v}) \mid$.

Lemma 3.1 implies that every $\mathcal{P}_{1 b}$-identity of $S$ can be derived from some almostlinear and block-balanced identities of $S$. Therefore, every identity of $S$ can be derived from some almost-linear and block-balanced identities of $S$.

Claim 6. Every block-balanced identity of $S$ can be derived from some block-balanced identities of $S$ with two non-linear variables.

Proof. We assign a Type to each pair $\{c, d\} \subseteq \operatorname{OccSet}(\mathbf{u})$ of occurrences of distinct non-linear variables $x \neq y$ in a word $\mathbf{u}$ as follows. If $\{c, d\}=\left\{\right.$ last $\left._{\mathbf{u}} x,{ }_{1 \mathbf{u}} y\right\}$ then we say that $\{c, d\}$ is of Type 2. Otherwise, $\{c, d\}$ is of Type 1 .

Let $\Delta$ be the set of all block-balanced identities of $S$ with two non-linear variables. Let $\mathbf{u} \approx \mathbf{v}$ be a block-balanced identity of $S$ and $\{c, d\} \subseteq \operatorname{OccSet}(\mathbf{u})$ be a critical pair in $\mathbf{u} \approx \mathbf{v}$. Suppose that $\{c, d\}$ is of Type 1 . Then by using an identity from $\left\{\sigma_{1}, \sigma_{2}\right\}^{\delta}$ we swap $c$ and $d$ in $\mathbf{u}$ and obtain a word $\mathbf{w}$. Evidently, the word $\mathbf{w}$ satisfies all the requirements of Lemma 3.4.

Now suppose that $\{c, d\}$ is of Type 2. Then $\{c, d\}=\left\{\right.$ lastu $\left._{\mathbf{u}} x,{ }_{1 \mathbf{u}} y\right\}$ for some variables $x \neq y$. Four cases are possible.

Case 1: There are no linear letters in $\mathbf{u}$ between ${ }_{1 \mathbf{u}} x$ and last $\mathbf{u} y$.

Case 2: There are no linear letters in $\mathbf{u}$ between ${ }_{1 \mathbf{u}} y$ and ${ }_{\text {last }} y$ but there is a linear letter between $1 \mathbf{u} x$ and lastu $x$.

Case 3: There are no linear letters in $\mathbf{u}$ between ${ }_{1 \mathbf{u}} x$ and ${ }_{\text {last } \mathbf{u}} x$ but there is a linear letter between ${ }_{1 \mathbf{u}} y$ and last $\mathbf{u} y$.

Case 4: There is a linear letter in $\mathbf{u}$ between ${ }_{1 \mathbf{u}} x$ and ${ }_{\text {last }} x$ and there is a linear letter between $1 \mathbf{u} y$ and lastu $y$.

All cases are similar. We consider only Case 2. Let $\mathbf{A}$ be an almost-linear word such that $\mathbf{u}(x, \operatorname{Lin}(\mathbf{u}))=\mathbf{A} x$. If $\operatorname{occ}_{\mathbf{u}}(y)=k$ then by Condition (ii), $S$ satisfies the identity $\mathbf{A} x y^{c} t y^{k-c} \approx \mathbf{A} y x y^{c-1} t y^{k-c}$ for some $0<c<k$. In this case, by using $\left\{\sigma_{1}, \sigma_{2}\right\}^{\delta}$ we obtain a word $\mathbf{r}$ so that all the elements of OccSet $(\mathbf{r})$ which are in the set $\left\{\operatorname{lastr}_{\mathbf{r}} x,{ }_{1 \mathbf{r}} y,{ }_{2 \mathbf{r}} y, \ldots,{ }_{\mathrm{r}} y\right\}$ and all the elements of $\operatorname{OccSet}(\mathbf{r})$ which are in the set $\{(c+1) \mathbf{r} y,(c+2) \mathbf{r} y, \ldots, k \mathbf{r} y\}$ are collected together. After that, we apply the identity $\mathbf{A} x y^{c} t y^{k-c} \approx \mathbf{A} y x y^{c-1} t y^{k-c}$ to $\mathbf{r}$ and obtain a word $\mathbf{w}$. It is easy to see that the word $\mathbf{w}$ satisfies all the requirements of Lemma 3.4.

In view of Lemma 4.1, every block-balanced identity with two non-linear variables $x \neq y$ which is not a consequence of $\left\{\sigma_{1}, \sigma_{2}\right\}^{\delta}$ is equivalent modulo $\left\{\sigma_{1}, \sigma_{2}\right\}^{\delta}$ to $x^{\alpha} y^{\beta} \approx x y^{\beta} x^{\alpha-1}$ for some $\alpha, \beta>1$ or to an identity of the following form: 


$$
\begin{gathered}
x^{\alpha_{0}} t_{1} x^{\alpha_{1}} t_{2} \ldots x^{\alpha_{n-1}} t_{n} x^{\alpha_{n}} y^{\beta_{m}} t_{n+1} y^{\beta_{m-1}} \ldots y^{\beta_{3}} t_{n+m-1} y^{\beta_{1}} t_{n+m} y^{\beta_{0}} \approx \\
x^{\alpha_{0}} t_{1} x^{\alpha_{1}} t_{2} \ldots x^{\alpha_{n-1}} t_{n} y^{\beta_{m}} x^{\alpha_{n}} t_{n+1} y^{\beta_{m-1}} \ldots y^{\beta_{3}} t_{n+m-1} y^{\beta_{1}} t_{n+m} y^{\beta_{0}}
\end{gathered}
$$

where $n, m, \alpha_{n}, \beta_{m}>0$ and $\alpha_{0}, \beta_{0}, \ldots, \alpha_{n-1}, \beta_{m-1} \geq 0$.

By using the same arguments as in the proof of Proposition 5.7 in [9] (see Lemma 2.3 above) one can show that in the presence of $\left\{\sigma_{1}, \sigma_{2}\right\}^{\delta}$, every set of identities of this form can be derived from a finite subset.

Now Claims 5 and 6 and the result of Volkov (Lemma 2.2) imply that the monoid $S$ is finitely based by some almost-linear identities and by some block-balanced identities with two non-linear variables.

Corollary 4.11. Suppose that each word in $W$ is either almost-linear or of the form $a_{1}^{\alpha_{1}} \ldots a_{m}^{\alpha_{m}}$ for some distinct letters $a_{1}, \ldots, a_{m}$ and positive numbers $\alpha_{1}, \ldots, \alpha_{m}$. Then the monoid $S(W)$ is finitely based.

Proof. First notice that $S(W)$ is equationally equivalent to a monoid $S\left(W^{\prime}\right)$ where $W^{\prime}$ consists of all almost-linear words in $W$ and of all subwords of words in $W$ of the form $a^{\alpha} b^{\beta}$. Indeed, each word in $W^{\prime}$ is an isoterm for $S(W)$. Conversely, each word $\mathbf{u} \in W$ is an isoterm for $S\left(W^{\prime}\right)$ because each adjacent pair of variables in $\mathbf{u}$ is stable in $\mathbf{u}$ with respect to $W^{\prime}$ (see Fact 3.4 in [13]).

It is easy to see that $S\left(W^{\prime}\right)$ satisfies all conditions of Theorem 4.10. (One can also use Theorem 3.1 in [12].) Consequently, the monoid $S(W)$ is finitely based as well.

Theorem 4.12. Let $S$ be a monoid such that $S \models\left\{\sigma_{1}, \sigma_{2}\right\}$. Suppose also that for some $m>0$ the word $x^{m} y^{m}$ is an isoterm for $S$ and for some $0<d \leq m$, $S$ satisfies either $x^{m+1-d} t x^{d} y t y \approx x^{m+1-d} t x^{d-1} y x t y$ or $x t x y^{d} t y^{m+1-d} \approx x t y x y^{d-1} t y^{m+1-d}$. If $m>1$ then we suppose that for each $1<k \leq m$, S satisfies each of the following dual conditions:

(i) If for some almost-linear word $\mathbf{A} x$ with $\operatorname{occ}_{\mathbf{A}}(x)>0$ the pair $\{x, y\}$ is bunstable in $\mathbf{A} x y^{k}$ with respect to $S$ then for some $0<c<k, S$ satisfies the identity $\mathbf{A} x y^{c} t y^{k-c} \approx \mathbf{A} y x y^{c-1} t y^{k-c}$;

(ii) If for some almost-linear word $y \mathbf{B}$ with $\operatorname{occ}_{\mathbf{B}}(y)>0$ the pair $\{x, y\}$ is bunstable in $x^{k} y \mathbf{B}$ with respect to $S$ then for some $0<p<k$, S satisfies the identity $x^{k-p} t x^{p} y \mathbf{B} \approx x^{k-p} t x^{p-1} y x \mathbf{B}$.

Then $S$ is finitely based by some almost-linear identities and by some blockbalanced identities with two non-linear variables.

Proof. If $m=1$ then $S \models \sigma_{\mu}$ and by Theorem 3.3, the monoid $S$ is finitely based by some almost-linear identities and by $\left\{\sigma_{1}, \sigma_{2}, \sigma_{\mu}\right\}^{\delta}$. So, we may assume that $m>1$ and, since all conditions are symmetric, we may also assume that for some $0<d \leq m, S$ satisfies $x^{m+1-d} t x^{d} y t y \approx x^{m+1-d} t x^{d-1} y x t y$.

Claim 7. Every identity of $S$ can be derived from some almost-linear and blockbalanced identities of $S$. 
Proof. Similar to the proof of Claim 5 .

Claim 8. Every block-balanced identity of $S$ can be derived from some block-balanced identities of $S$ with two non-linear variables.

Proof. We assign a Type to each pair $\{c, d\} \subseteq \operatorname{OccSet}(\mathbf{u})$ of occurrences of distinct non-linear variables in a word $\mathbf{u}$ as follows. If $\{c, d\}$ is not of the form $\left\{\right.$ last $\left._{\mathbf{u}} x,{ }_{1 \mathbf{u}} y\right\}$ for any non-linear variables $x \neq y$ then we say that $\{c, d\}$ is of Type 1 . If $\{c, d\}=$ $\left\{\right.$ last $\left._{\mathbf{u}} x,{ }_{1 \mathbf{u}} y\right\}$ for some variables $x \neq y$ with $2 \leq \operatorname{occ}_{\mathbf{u}}(x) \leq m, \operatorname{occ}_{\mathbf{u}}(y) \geq 2$ and there is no linear letter in $\mathbf{u}$ between ${ }_{1 \mathbf{u}} x$ and ${ }_{\text {last }} y$ then we say that $\{c, d\}$ is of Type 3 . Otherwise, we say that $\{c, d\}$ is of Type 2 .

Let $\mathbf{u} \approx \mathbf{v}$ be a block-balanced identity of $S$ and $\{c, d\} \subseteq \operatorname{OccSet}(\mathbf{u})$ be a critical pair in $\mathbf{u} \approx \mathbf{v}$. Suppose that $\{c, d\}$ is of Type 1 . Then by using an identity from $\left\{\sigma_{1}, \sigma_{2}\right\}^{\delta}$ we swap $c$ and $d$ in $\mathbf{u}$ and obtain a word $\mathbf{w}$. Evidently, the word $\mathbf{w}$ satisfies all the requirements of Lemma 3.4.

Now suppose that $\{c, d\}$ is of Type 2. Then $\{c, d\}=\left\{\right.$ lastu $\left._{\mathbf{u}} x,{ }_{1 \mathbf{u}} y\right\}$ for some variables $x \neq y$.

Case 1: $\operatorname{occ}_{\mathbf{u}}(x)=n>m$ and there is no linear letter in $\mathbf{u}$ between $1 \mathbf{u} x$ and lastuy.

In this case, by using $\left\{\sigma_{1}, \sigma_{2}\right\}^{\delta}$ we obtain a word $\mathbf{f}$ so that all the elements of $\operatorname{OccSet}(\mathbf{f})$ which are in the set $\left\{{ }_{1 \mathbf{f}} x,{ }_{2 \mathbf{f}} x, \ldots,{ }_{(n-d) \mathbf{f}} x\right\}$ and all the elements of $\operatorname{OccSet}(\mathbf{f})$ which are in the set $\left\{(n-d+1) \mathbf{f} x, \ldots, n \mathbf{f} x,{ }_{1 \mathbf{f}} y\right\}$ are collected together. After that by using an identity in $\left\{x^{m+1-d} t x^{d} y t y \approx x^{m+1-d} t x^{d-1} y x t y\right\}^{\delta}$ we swap $c$ and $d$ in $\mathbf{f}$ and obtain a word $\mathbf{w}$. It is easy to see that the word $\mathbf{w}$ satisfies all the requirements of Lemma 3.4.

Case 2: there is a linear letter in $\mathbf{u}$ between ${ }_{1 \mathbf{u}} x$ and ${ }_{\text {last } \mathbf{u}} y$.

We handle this case exactly as Cases 2, 3 and 4 in the proof of Theorem 4.10.

Finally, suppose that $\{c, d\}$ is of Type 3. Then $\{c, d\}=\left\{\right.$ lastu $\left._{\mathbf{u}} x,{ }_{1 \mathbf{u}} y\right\}, \operatorname{occ}_{\mathbf{u}}(x)=$ $n \leq m$ and there is no linear letter in $\mathbf{u}$ between ${ }_{1 \mathbf{u}} x$ and ${ }_{\text {last }} y$.

Denote $\operatorname{occ}_{\mathbf{u}}(y)=k$. Since the word $x^{m} y^{m}$ is an isoterm for $S$, we have $k>$ $m$. First, we collect all occurrences of $y$ together as follows. By using $\left\{\sigma_{1}, \sigma_{2}\right\}^{\delta}$ we obtain a word $\mathbf{r}$ such that all the elements of $\operatorname{OccSet}(\mathbf{u})$ which are in the set $\left\{\right.$ last $\left._{\mathbf{r}} x,{ }_{1 \mathbf{r}} y,{ }_{2 \mathbf{r}} y, \ldots,(k-1) \mathbf{r} y\right\}$ are collected together. If ${ }_{(k-1) \mathbf{r}} y$ and ${ }_{k \mathbf{r}} y$ are not adjacent in $\mathbf{r}$ then one can find an occurrence $p$ of some non-linear variable $z \notin\{x, y\}$ such that $p \ll_{\mathbf{r}}\left(k_{\mathbf{r}} y\right)$. If $p$ is not the first occurrence of $z$ then by using an identity in $\left\{\sigma_{2}\right\}^{\delta}$, we obtain a word $\mathbf{s}$ such that $\left.{ }_{k \mathbf{s}} y\right) \ll_{\mathbf{s}} p$. If $p$ is the first occurrence of $z$ then first, by using $\left\{\sigma_{1}, \sigma_{2}\right\}^{\delta}$ we obtain a word $\mathbf{q}$ such that all the elements of $\operatorname{OccSet}(\mathbf{q})$ which are in the set $\left\{(k-d+1) \mathbf{q} y, \ldots,(k-1) \mathbf{q} y, p, k_{k} y\right\}$ are collected together. After that, by using an identity in $\left\{y^{m+1-d} t y^{d} z t z \approx y^{m+1-d} t y^{d-1} z y t z\right\}^{\delta}$, we obtain a word $\mathbf{s}$ such that $\left.{ }_{k \mathbf{s}} y\right) \ll_{\mathbf{s}} p$. And so on. Eventually, we obtain a word $\mathbf{t}$ such that all the elements of OccSet(t) which are in the set $\left\{{ }_{\text {last }} x,_{1 \mathbf{t}} y,{ }_{2 \mathbf{t}} y, \ldots,{ }_{k \mathbf{t}} y\right\}$ are collected together.

Now by Condition (ii), $S$ satisfies the identity $x^{n-p} t x^{p} y^{k} \approx x^{n-p} t x^{p-1} y x y^{k-1}$ for some $0<p<n$. By using $\left\{\sigma_{1}, \sigma_{2}\right\}^{\delta}$ we obtain a word e so that all the elements 
of $\operatorname{OccSet}(\mathbf{e})$ which are in the set $\left\{{ }_{1} x,{ }_{2} \mathbf{e} x, \ldots,(n-p) \mathbf{e} x\right\}$ and all the elements of $\operatorname{OccSet}(\mathbf{e})$ which are in the set $\left\{(n-p+1) \mathbf{e} x, \ldots,{ }_{n} \mathbf{e} x, 1 \mathbf{e} y\right\}$ are collected together. After that by using $x^{n-p} t x^{p} y^{k} \approx x^{n-p} t x^{p-1} y x y^{k-1}$ we swap $c$ and $d$ in e and obtain a word $\mathbf{w}$. It is easy to see that the word $\mathbf{w}$ satisfies all the requirements of Lemma 3.4.

The rest is similar to the proof of Theorem 4.10.

Example 4.13. (i) The monoid $S\left(\left\{a^{3} b^{2}, a^{2} b^{3}\right\}\right)$ is finitely based by Theorem 4.10 but fails Theorem 4.12.

(ii) The monoid $S\left(\left\{a^{2} t_{1} a^{2} b^{2} t_{2} b\right\}\right)$ is finitely based by Theorem 4.12 but fails Theorem 4.10 .

(iii) The monoid $S\left(\left\{a^{2} t_{1} a^{2} b^{2} t_{2} b^{2}\right\}\right)$ is non-finitely based.

Proof. First notice that each of these monoids satisfies $\left\{\sigma_{1}, \sigma_{2}\right\}$ by Fact 4.7 .

(i) The word $a^{2} b^{2}$ is an isoterm for $S\left(\left\{a^{3} b^{2}, a^{2} b^{3}\right\}\right)$, but $S\left(\left\{a^{3} b^{2}, a^{2} b^{3}\right\}\right)$ satisfies none of the following identities $\{x x t x y t y \approx x x t y x t y$, xtxxyty $\approx$ xtxyxty, xtxytyy $\approx$ xtyxtyy, xtxyyty $\approx$ xtyxyty $\}$. So, Theorem 4.12 is not applicable here. On the other hand, $S\left(\left\{a^{3} b^{2}, a^{2} b^{3}\right\}\right)$ is finitely based by Corollary 4.11.

(ii) Notice that $S\left(\left\{a^{2} t_{1} a^{2} b^{2} t_{2} b\right\}\right) \models x^{3} y^{2} \approx y^{2} x^{3}$. So, the word $a^{3} b^{2}$ is not an isoterm for $S\left(\left\{a^{2} t_{1} a^{2} b^{2} t_{2} b\right\}\right)$. But each of the words $\left\{a^{2} t a b t b, a t a^{2} b t b\right\}$ is an isoterm for $S\left(\left\{a^{2} t_{1} a^{2} b^{2} t_{2} b\right\}\right)$. So, Theorem 4.10 is not applicable here.

On the other hand, the word $a^{2} b^{2}$ is an isoterm for $S$ and $S \models x t x y t y y \approx x t y x t y y$. Since the words $\{a t a b b, a a b t b\}$ are isoterms for $S$, Conditions (i) and (ii) of Theorem 4.12 are trivially satisfied. So, $S$ is finitely based by Theorem 4.12 ,

(iii) The monoid $S=S\left(\left\{a^{2} t_{1} a^{2} b^{2} t_{2} b^{2}\right\}\right)$ is non-finitely based by Theorem 4.4 (row $8)$ in [13. This is because the words $\left\{a^{2} b^{2}\right.$, atabtbb, atabbtb, ataabtb, aatabtb $\}$ are isoterms for $S$ and for each $n>1$ we have $S \models y t x x y p_{1}^{2} \ldots p_{n}^{2} z x t z \approx y t x x x y p_{1}^{2} \ldots p_{n}^{2} z t z$.

\section{Some finitely based subvarieties of $\operatorname{var}\left\{\sigma_{\mu}\right\}$}

We say that a word $\mathbf{u}$ is compact if all occurrences of all non-linear variables in $\mathbf{u}$ are collected together in each block of $\mathbf{u}$. For example, the word $x_{x y t_{1}} y y y t_{2} x$ is compact because it is $x x$-compact and $y y$-compact. The word $x y y x$ is not compact. The next lemma is needed only to prove Theorem 5.3.

Lemma 5.1. Every 2-limited word is equivalent to a compact word modulo $\left\{\sigma_{\mu}\right.$, yxxty $\approx$ xxyty $\}^{\delta}$.

Proof. Let $\mathbf{u}$ be a 2 -limited word. We say that a 2 -occurring variable is an $\mathcal{L}$-variable in $\mathbf{u}$ if there are no linear letters between ${ }_{1 \mathbf{u}} x$ and ${ }_{2 \mathbf{u}} x$. We use $\mathfrak{Q}(\mathbf{u}, x)$ to denote the set of all $\mathcal{L}$-variables $y \neq x$ such that both occurrences of $y$ are between ${ }_{1 \mathbf{u}} x$ and ${ }_{2 \mathbf{u}} x$. We use $Y(\mathbf{u}, x)$ to denote the set of all occurrences of variables between ${ }_{1 \mathbf{u}} x$ and ${ }_{2 \mathbf{u}} x$. If $x$ is an $\mathcal{L}$-variable and $\mathfrak{Q}(\mathbf{u}, x)=\left\{z_{1}, \ldots, z_{m}\right\}$ for some $m \geq 0$, then $Y(\mathbf{u}, x)=Y_{1} \cup Y_{2} \cup\left\{{ }_{1 \mathbf{u}} z_{1},{ }_{2} \mathbf{u} z_{1}, \ldots,{ }_{1} \mathbf{u} z_{m},{ }_{2} \mathbf{u} z_{m}\right\}$ where each element of $Y_{1}$ is the first 
occurrence of some variable in $\mathbf{u}$ and each element of $Y_{2}$ is the second occurrence of some variable in $\mathbf{u}$. The desired statement is an immediate consequence of the following.

Claim 9. Every 2-limited word $\mathbf{u}$ is equivalent modulo $\left\{\sigma_{\mu}, \text { yxxty } \approx \text { xxyty }\right\}^{\delta}$ to a word $\mathbf{p}$ with the property that for each $m \geq 0$ and for each $\mathcal{L}$-variable $x$ with $|\mathfrak{Q}(\mathbf{u}, x)| \leq m$ each of the following is true:

(i) $1 \mathbf{p} x \ll_{\mathbf{p}} \mathbf{p} x$

(ii) for each $c \in \operatorname{OccSet}(\mathbf{u})$ we have $c<_{\mathbf{p} 1 \mathbf{p}} x$ if $c<_{\mathbf{u} 1 \mathbf{u}} x$;

(iii) for each $c \in \operatorname{OccSet}(\mathbf{u})$ we have ${ }_{2 \mathbf{p}} x<_{\mathbf{p}} c$ if ${ }_{2 \mathbf{u}} x<_{\mathbf{u}} c$.

Proof. First, we prove the statement for $m=0$. Let $x$ be a $\mathcal{L}$-variable in $\mathbf{u}$ such that the set $\mathfrak{Q}(\mathbf{u}, x)$ is empty. Then $Y(\mathbf{u}, x)=Y_{1} \cup Y_{2}$. If $q^{\prime}$ is the smallest in order $<_{\mathbf{u}}$ element in $Y_{2}$, then by using the identities in $\left\{\sigma_{\mu}\right\}^{\delta}$ and commuting the adjacent occurrences of variables, we move $q^{\prime}$ to the left until we obtain a word $\mathbf{s}_{\mathbf{1}}$ so that $q^{\prime} \ll_{\mathbf{s}_{1}{ }_{1} \mathbf{s}_{\mathbf{1}}} x$. And so on. After repeating this $k=\left|Y_{2}\right|$ times, we obtain a word $\mathbf{s}_{\mathbf{k}}$ so that each occurrence of each variable between ${ }_{1} \mathbf{s}_{\mathbf{k}} x$ and $2 \mathbf{s}_{\mathbf{k}} x$ is the first occurrence of this variable. Now by using the identities in $\left\{\sigma_{\mu}\right\}^{\delta}$ and commuting the adjacent occurrences of variables, we move ${ }_{2 \mathbf{s}_{\mathbf{k}}} x$ to the left until we obtain a word $\mathbf{r}_{\mathbf{1}}$ so that ${ }_{1} \mathbf{r}_{1} x \ll_{\mathbf{r}_{1}} 2 \mathbf{r}_{1} x$. Since we only "push out" the elements of OccSet(u) which are between ${ }_{1 \mathbf{u}} x$ and ${ }_{2 \mathbf{u}} x$, the word $\mathbf{r}_{\mathbf{1}}$ satisfies Properties (ii)-(iii) as well.

If $z \neq x$ is another $\mathcal{L}$-variable in $\mathbf{u}$ such that the set $\mathfrak{Q}(\mathbf{u}, z)$ is empty, then by repeating the same procedure, we obtain a word $\mathbf{r}_{2}$ so that ${ }_{1 \mathbf{r}_{2}} x \ll_{\mathbf{r}_{2}} 2 \mathbf{r}_{\mathbf{2}} x,{ }_{1 \mathbf{r}_{2}} z \ll_{\mathbf{r}_{2}}$ $2 \mathbf{r}_{2} z$ and Properties (ii)-(iii) are satisfied for $x$ and $z$. And so on. Thus, the base of induction is established.

Let $x$ be an $\mathcal{L}$-variable in $\mathbf{u}$ with $\mathfrak{Q}(\mathbf{u}, x)=\left\{z_{1}, \ldots, z_{m}\right\}$. By our induction hypothesis, the word $\mathbf{u}$ is equivalent modulo $\left\{\sigma_{\mu}, \text { yxxty } \approx x x y t y\right\}^{\delta}$ to a word $\mathbf{p}$ with the property that for each $i=1, \ldots, m$ we have ${ }_{1 \mathbf{p}} x<_{\mathbf{p} 1 \mathbf{p}} z_{i} \ll_{\mathbf{p} 2 \mathbf{p}} z_{i}<_{\mathbf{p} 2 \mathbf{p}} x$. If $q^{\prime}$ is the smallest in order $<_{\mathbf{p}}$ element in $Y_{2} \cup\left\{1 \mathbf{p} z_{1}, 2_{\mathbf{p}} z_{1}, \ldots,{ }_{1} z_{m},{ }_{2} \mathbf{p} z_{m}\right\}$, then we do the following. If $q^{\prime} \in Y_{2}$ then by using the identities in $\left\{\sigma_{\mu}\right\}^{\delta}$ and commuting the adjacent occurrences of variables, we move $q^{\prime}$ to the left until we obtain a word $\mathbf{s}_{\mathbf{1}}$ so that $q^{\prime} \ll_{\mathbf{s}_{1}{ }_{1} \mathbf{s}_{1}} x$. If $q^{\prime}={ }_{1 \mathbf{p}} z_{i}$ for some $i=1, \ldots, m$, then by using the identities in $\{y x x t y \approx x x y t y\}^{\delta}$, we move $\left({ }_{1} z_{i}\right)\left({ }_{2} \mathbf{p} z_{i}\right)$ to the left until we obtain a word $\mathbf{s}_{\mathbf{1}}$ so that $\left({ }_{1 \mathbf{s}_{\mathbf{1}}} z_{i}\right) \ll_{\mathbf{s}_{\mathbf{1}}}\left({ }_{2 \mathbf{s}_{\mathbf{1}}} z_{i}\right) \ll_{\mathbf{s}_{\mathbf{1}}}{ }_{1 \mathbf{s}_{\mathbf{1}}} x$. And so on. After repeating this $k=\left|Y_{2}\right|+m$ times, we obtain a word $\mathbf{s}_{\mathbf{k}}$ such that each occurrence of each variable between $1 \mathbf{s}_{\mathbf{k}} x$ and ${ }_{2 \mathbf{s}_{\mathbf{k}}} x$ is the first occurrence of this variable. Now by using the identity $\sigma_{\mu}$ and commuting the adjacent occurrences of variables, we move ${ }_{2 \mathbf{s}_{\mathbf{k}}} x$ to the left until we obtain a word $\mathbf{r}_{1}$ such that $1 \mathbf{r}_{1} x \ll_{\mathbf{r}_{1} 2 \mathbf{r}_{1}} x$.

If $z \neq x$ is another $\mathcal{L}$-variable in $\mathbf{u}$ with $\mathfrak{Q}(\mathbf{u}, x)=m$, then we repeat the same procedure and obtain a word $\mathbf{r}_{2}$ so that ${ }_{1 \mathbf{r}_{2}} x \ll_{\mathbf{r}_{2}} 2 \mathbf{r}_{2} x, 1_{\mathbf{r}_{2}} z \ll_{\mathbf{r}_{2}} 2 \mathbf{r}_{2} z$ and Properties (ii)-(iii) are satisfied for $x$ and $z$. And so on. Thus, the step of induction is established. 
Fact 5.2. (i) If the word xytyx is an isoterm for a monoid $S$ then the words xyztxzy and yzxtzyx can form an identity of $S$ only with each other.

(ii) The word xyztxzy is an isoterm for a monoid $S$ if and only if the word yzxtzyx is an isoterm for $S$.

Proof. (i) If $S$ satisfies an identity $x y z t x z y \approx \mathbf{u}$ then we have $\mathbf{u}(y, z, t)=y z t z y$. If $\mathbf{u} \neq x y z t x z y$ then the only possibility for $\mathbf{u}$ is $y z x t z y x$.

Part (ii) immediately follows from part (i).

We say that an identity $\mathbf{u} \approx \mathbf{v}$ is a compact identity if both $\mathbf{u}$ and $\mathbf{v}$ are compact words. Part (i) of the following statement generalizes Theorem 3.2 in [7] which says that the monoid $S(\{a b t a b, a b t b a\})$ is finitely based.

Theorem 5.3. Let $S$ be a monoid such that $S \models\left\{t_{1} x t_{2} x t_{3} x \approx x^{3} t_{1} t_{2} t_{3}, x^{3} \approx\right.$ $x^{4}, \sigma_{\mu}, y x x t y \approx$ xxyty $\}=\Omega$. Suppose also that $S$ satisfies one of the following conditions:

(i) both words xytyx and xytxy are isoterms for $S$;

(ii) the word xyztxzy is an isoterm for $S$.

Then $S$ is finitely based by a subset of $\Omega \cup\{y t y x x \approx y t x x y, x x t \approx t x x, x y t x y \approx$ yxtyx, $\left.x^{2} \approx x^{3}\right\}^{\delta}$.

Proof. Let $\Delta$ denote the subset of $\left\{\sigma_{\mu}, y x x t y \approx x x y t y, y t y x x \approx y t x x y, x y t x y \approx\right.$ $y x t y x, x x t \approx t x x\}^{\delta}$ satisfied by $S$. We use Lemma 3.4 to show that every 2-limited compact identity of $S$ is a consequence of $\Delta$.

We assign a Type to each pair $\{c, d\} \subseteq \operatorname{OccSet}(\mathbf{u})$ of occurrences of distinct variables $x \neq y$ with $\operatorname{occ}_{\mathbf{u}}(x) \leq 2$ and $\operatorname{occ}_{\mathbf{u}}(y) \leq 2$ as follows. If both $x$ and $y$ are 2-occurring, $\{c, d\}=\left\{{ }_{1 \mathbf{u}} x,{ }_{1 \mathbf{u}} y\right\}$ or $\{c, d\}=\left\{{ }_{2 \mathbf{u}} x,{ }_{2 \mathbf{u}} y\right\}$ and there is a linear letter (possibly the same) between ${ }_{1 \mathbf{u}} x$ and ${ }_{2 \mathbf{u}} x$ and between ${ }_{1 \mathbf{u}} y$ and ${ }_{2 \mathbf{u}} y$ then we say that $\{c, d\}$ is of Type 2. Otherwise, $\{c, d\}$ is of Type 1 .

Let $\mathbf{u} \approx \mathbf{v}$ be a 2-limited compact identity of $S$ and $\{c, d\} \subseteq \operatorname{OccSet}(\mathbf{u})$ be a critical pair in $\mathbf{u} \approx \mathbf{v}$. Suppose that $\{c, d\}$ is of Type 1 .

First assume that, say $c$ is the only occurrence of a linear variable $t$ in $\mathbf{u}$. Then, since the word $x t x$ is an isoterm for $S, d$ must be an occurrence of a 2-occurring variable $x$ and $\mathbf{u}(x, t) \approx \mathbf{v}(x, t)$ is the following identity: $x x t \approx t x x$. Since ${ }_{1 \mathbf{u}} x \ll_{\mathbf{u}}$ $2 \mathbf{u} x$, we can apply $x x t \approx t x x$ to $\mathbf{u}$ and obtain the word $\mathbf{w}$. Evidently, the word $\mathbf{w}$ satisfies all the requirements of Lemma 3.4.

Next assume that $\{c, d\}=\left\{{ }_{1 \mathbf{u}} x,{ }_{2 \mathbf{u}} y\right\}$ for some 2-occurring variables $x$ and $y$. If there are linear letters between ${ }_{1 \mathbf{u}} x$ and ${ }_{2 \mathbf{u}} x$ and between ${ }_{1 \mathbf{u}} y$ and ${ }_{2 \mathbf{u}} y$ then by using an identity from $\left\{\sigma_{\mu}\right\}^{\delta}$ we swap $c$ and $d$ in $\mathbf{u}$ and obtain a word $\mathbf{w}$. Otherwise, we swap $c$ and $d$ in $\mathbf{u}$ by using the identity $x x t \approx t x x$. In any case the resulting word w satisfies all the requirements of Lemma 3.4.

Now assume that $c={ }_{1 \mathbf{u}} x \ll_{\mathbf{u}} 1 \mathbf{u} y=d$ for some 2-occurring variables $x$ and $y$. Let $a$ denote the smallest in order $<_{\mathbf{u}}$ element of the set $\left\{2_{\mathbf{u}} x,{ }_{2 \mathbf{u}} y\right\}$. Since $\{c, d\}$ is of Type 1 , there is no linear letter between $1 \mathbf{u} y$ and $a$. Since both $\mathbf{u}$ and $\mathbf{v}$ are compact words, we have that $a={ }_{2 \mathbf{u}} y,\left({ }_{1 \mathbf{u}} x\right) \ll_{\mathbf{u}}\left({ }_{1 \mathbf{u}} y\right) \ll_{\mathbf{u}}\left({ }_{2 \mathbf{u}} y\right)$ and $\left({ }_{1 \mathbf{v}} y\right) \ll_{\mathbf{v}}\left({ }_{2 \mathbf{v}} y\right)$. We use the 
identity $x y y t x \approx$ yyxt $x$ and obtain the word $\mathbf{w}$ so that $\left({ }_{1 \mathrm{w}} y\right) \ll_{\mathbf{w}}\left({ }_{2 \mathrm{w}} y\right) \ll_{\mathbf{w}}\left({ }_{1 \mathrm{w}} x\right)$. It is easy to check that the word $\mathbf{w}$ satisfies all the requirements of Lemma 3.4.

Finally, assume that $c={ }_{2 \mathbf{u}} x \ll_{\mathbf{u}} 2 \mathbf{u} y=d$ for some 2-occurring variables $x$ and $y$. Let $b$ denote the largest in order $<_{\mathbf{u}}$ element of the set $\left\{1_{\mathbf{u}} x,{ }_{1 \mathbf{u}} y\right\}$. Since $\{c, d\}$ is of Type 1 , there is no linear letter between $b$ and ${ }_{2 \mathbf{u}} x$. Since $\mathbf{u}$ is a compact word, we have that $b={ }_{1 \mathbf{u}} x,\left({ }_{1 \mathbf{u}} x\right) \ll_{\mathbf{u}}\left({ }_{2 \mathbf{u}} x\right) \ll_{\mathbf{u}}(2 \mathbf{u} y),\left({ }_{1 \mathbf{v}} x\right) \ll_{\mathbf{v}}(2 \mathbf{v} x)$ and there is a linear letter between ${ }_{1 \mathbf{u}} y$ and ${ }_{1 \mathbf{u}} x$. We apply the identity $y \operatorname{tx} x y=\mathbf{u}(x, y, t) \approx \mathbf{v}(x, y, t)=$ $y \operatorname{tyx} x$ to $\mathbf{u}$ and obtain a word $\mathbf{w}$ which satisfies all the requirements of Lemma 3.4.

If $S$ satisfies Condition (i) which says that both words xytyx and xytxy are isoterms for $S$, then the identity $\mathbf{u} \approx \mathbf{v}$ does not have any unstable pairs of Type 2 and we are done.

Let us suppose that $S$ satisfies Condition (ii) which says that the word $x y z t x z y$ is an isoterm for $S$. If $\{c, d\}$ is of Type 2 , then $\{c, d\}=\left\{{ }_{1 \mathbf{u}} x,{ }_{1} \mathbf{u} y\right\}$ or $\{c, d\}=\{2 \mathbf{u} x, 2 \mathbf{u} y\}$ for some 2-occurring variables $x \neq y$ and there is a linear letter between $1 \mathbf{u} x$ and ${ }_{2} \mathbf{u}$ and between ${ }_{1 \mathbf{u}} y$ and ${ }_{2 \mathbf{u}} y$. Since the word $x y t y x$ is an isoterm for $S$, for some letter $t$ we have $\mathbf{u}(x, y, t)=x y t x y$ and $\mathbf{v}(x, y, t)=y x t y x$.

In view of the symmetry, without loss of generality, we may assume that $c=$ ${ }_{1 \mathbf{u}} x \ll_{\mathbf{u}} 1 \mathbf{u} y=d$. Since the word $x y t_{1} x t_{2} y$ is an isoterm for $S$, there is no linear letter in $\mathbf{u}$ between ${ }_{2 \mathbf{u}} x$ and $2 \mathbf{u} y$.

Claim 10. If for some variable $z$ we have ${ }_{2 \mathbf{u}} x<_{\mathbf{u} 2 \mathbf{u}} z<_{\mathbf{u}} 2 \mathbf{u} y$ then we have ${ }_{2 \mathbf{u}} x<_{\mathbf{u}}$ $1 \mathbf{u} z \ll_{\mathbf{u} 2 \mathbf{u}} z<_{\mathbf{u} 2 \mathbf{u}} y$.

Proof. If there is a linear letter between ${ }_{1 \mathbf{u}} z$ and ${ }_{2 \mathbf{u}} z$ then for some letter $t$ we have $\mathbf{u}(x, y, z, t)=x y z t x z y$ or $\mathbf{u}(x, y, z, t)=z x y t x z y$. But by Fact 5.2 , both these words are isoterms for $S$. The rest follows from the fact that $\mathbf{u}$ is a compact word.

We use $Y(\mathbf{u}, x, y)$ to denote the set of all occurrences of variables between $2 \mathbf{u} x$ and ${ }_{2 \mathbf{u}} y$. In view of Claim 10 we have $Y(\mathbf{u}, x, y)=Y_{1} \cup\left\{{ }_{1 \mathbf{u}} z_{1},{ }_{2 \mathbf{u}} z_{1}, \ldots,{ }_{1} z_{m}, 2 \mathbf{u} z_{m}\right\}$ where each element of $Y_{1}$ is the first occurrence of some variable in $\mathbf{u}$. If $m>0$ then it is easy to see that $S$ satisfies the identity $y t y x x \approx y t x x y$. Suppose that the set $Y(\mathbf{u}, x, y)$ is not empty and $q$ is the smallest in order $<_{\mathbf{u}}$ element in $Y(\mathbf{u}, x, y)$. If $q \in Y_{1}$, we use $\left\{\sigma_{\mu}\right\}^{\delta}$ and obtain a word $\mathbf{r}_{1}$ so that $q \ll_{\mathbf{r}_{1}} 2 \mathbf{r}_{1} x$. If $q$ is the first occurrence of $z_{i}$ for some $i=1, \ldots, m$, then we use ytyx $x \approx y t x x y$ and obtain a word $\mathbf{r}_{1}$ so that ${ }_{1 \mathbf{p}} z_{1} \ll_{\mathbf{r}_{1} 2 \mathbf{p}} z_{1} \ll_{\mathbf{r}_{1} 2 \mathbf{u}} x$. In any case we have $\left|Y\left(\mathbf{r}_{1}, x, y\right)\right|<|Y(\mathbf{u}, x, y)|$. And so on. After at most $|Y(\mathbf{u}, x, y)|$ steps we obtain a word $\mathbf{r}_{\mathbf{m}}$ so that the set $Y\left(\mathbf{r}_{\mathbf{m}}, x, y\right)$ is empty. This means that ${ }_{2 \mathbf{u}} x \ll_{\mathbf{r}_{\mathbf{m}}} 2 \mathbf{u} y$. Now we apply the identity $x y t x y \approx$ yxty $x$ to $\mathbf{r}_{\mathbf{m}}$ and obtain a word $\mathbf{w}$. It is easy to check that the word $\mathbf{w}$ satisfies all the requirements of Lemma 3.4.

So, every 2-limited compact identity of $S$ can be derived from $\Delta$. In view of Lemma 5.1, every 2-limited identity of $S$ can be derived from $\left\{\sigma_{\mu}, y x x t y \approx x x y t y\right\}^{\delta}$ and a compact identity of $S$. Finally, every identity of $S$ can be derived from a subset of $\left\{t_{1} x t_{2} x t_{3} x \approx x^{3} t_{1} t_{2} t_{3}, x^{3} \approx x^{4}, x^{2} \approx x^{3}\right\}^{\delta}$ and a 2-limited identity of $S$. Therefore, every identity of $S$ can be derived from a subset of $\Delta \cup\left\{t_{1} x t_{2} x t_{3} x \approx x^{3} t_{1} t_{2} t_{3}, x^{3} \approx\right.$ $\left.x^{4}, x^{2} \approx x^{3}\right\}^{\delta}=\Omega \cup\left\{y t y x x \approx y t x x y, x x t \approx t x x, x y t x y \approx y x t y x, x^{2} \approx x^{3}\right\}^{\delta}$. 
Example 5.4. The monoids $S($ abctacb) and $S$ (cbatbca) are equationally equivalent and finitely based.

Proof. These monoids are equationally equivalent by Fact 5.2 and finitely based by Theorem 5.3(ii).

According to [15], the monoid $S$ (abctacb) is not equationally equivalent to any monoid of the form $S(W)$ where $W$ is a set of words with two non-linear variables.

\section{Some derivation-stable properties of identities and a description of the equational theories for some varieties}

\begin{tabular}{|c|c|c|}
\hline defining formula for $\sim_{S}$ & generating monoid $S$ & basis of identities \\
\hline $\begin{array}{l}\mathbf{u} \approx \mathbf{v} \text { is regular: } \\
\operatorname{Cont}(\mathbf{u})=\operatorname{Cont}(\mathbf{v})\end{array}$ & two-element semilattice & $\{x \approx x x, x y \approx y x\}$ \\
\hline $\begin{array}{l}\mathbf{u} \approx \mathbf{v} \text { is balanced: } \\
\forall x \in \mathfrak{A}, \mathbf{u}(x)=\mathbf{v}(x)\end{array}$ & infinite cyclic semigroup & $\{x y \approx y x\}$ \\
\hline $\begin{array}{l}\mathbf{u} \approx \mathbf{v} \text { is block-balanced: } \\
\forall x \in \mathfrak{A}, \mathbf{u}(x, \operatorname{Lin}(\mathbf{u}))=\mathbf{v}(x, \operatorname{Lin}(\mathbf{v}))\end{array}$ & $\begin{array}{l}S\left(W_{A L}\right), W_{A L} \text { is the set } \\
\text { of all almost-linear words }\end{array}$ & $\left\{\sigma_{1}, \sigma_{\mu}, \sigma_{2}\right\}^{\delta}$ \\
\hline $\begin{array}{l}\mathbf{u} \approx \mathbf{v} \text { is } \mathcal{P}_{1,2} \text {-identity: } \operatorname{Lin}(\mathbf{u})=\operatorname{Lin}(\mathbf{v}), \\
\operatorname{Non}(\mathbf{u})=\operatorname{Non}(\mathbf{v}), \forall x, y \in \operatorname{Cont}(\mathbf{u}) \\
\left({ }_{\mathbf{u}} x\right)<_{\mathbf{u}}(\text { last } \mathbf{u}) \text { iff }\left({ }_{\mathbf{v}} x\right)<_{\mathbf{v}}(\text { last } \mathbf{v} y)\end{array}$ & $\begin{array}{l}\text { the monoid } A_{0}^{1} \\
\text { of order five }\end{array}$ & $\begin{array}{l}\left\{\sigma_{1}, \sigma_{2},\right. \\
\left.x t_{1} x t_{2} x \approx x t_{1} t_{2} x\right\}^{\delta}\end{array}$ \\
\hline $\begin{array}{l}\mathbf{u} \approx \mathbf{v} \text { is a block-balanced } \\
\mathcal{P}_{1,2^{2}} \text {-identity }\end{array}$ & $S\left(W_{A L} \cup\left\{a^{k} b^{k} \mid k>0\right\}\right)$ & $\left\{\sigma_{1}, \sigma_{2}\right\}^{\delta}$ \\
\hline $\begin{array}{l}\mathbf{u} \approx \mathbf{v} \text { is a } \mathcal{P}_{n} \text {-identity: } \\
\operatorname{Cont}(\mathbf{u})=\operatorname{Cont}(\mathbf{v}) \\
\mathbf{u}\left(\operatorname{Cont}_{n}(\mathbf{u})\right)=\mathbf{v}\left(\operatorname{Cont}_{n}(\mathbf{v})\right)\end{array}$ & $\begin{array}{l}S\left(W_{n}\right), W_{n} \text { is the set } \\
\text { of all } n \text {-limited words }\end{array}$ & $\begin{array}{l}\left\{t_{1} x t_{2} x t_{3} x \ldots t_{n+1} x \approx\right. \\
\approx x^{n+1} t_{1} t_{2} \ldots t_{n+1} \\
\left.x^{n+1} \approx x^{n+2}\right\}^{\delta}\end{array}$ \\
\hline $\begin{array}{l}\mathbf{u} \approx \mathbf{v} \text { is a } \mathcal{P}_{1} \text {-identity: } \\
\operatorname{Cont}(\mathbf{u})=\operatorname{Cont}(\mathbf{v}) \\
\mathbf{u}(\operatorname{Lin}(\mathbf{u}))=\mathbf{v}(\operatorname{Lin}(\mathbf{v}))\end{array}$ & $S(\{a b\})$ & $\begin{array}{l}\left\{x^{2} t \approx t x^{2} \approx x t x\right. \\
\left.x^{2} \approx x^{3}\right\}\end{array}$ \\
\hline
\end{tabular}

Table 1: Three ways to define a variety

We say that a property of identities $\mathcal{P}$ is derivation-stable if an identity $\tau$ satisfies property $\mathcal{P}$ whenever $\Sigma \vdash \tau$ and each identity in $\Sigma$ satisfies property $\mathcal{P}$. It is easy to check that such properties of identities as being a balanced identity, being a regular identity, being a $\mathcal{P}_{n}$-identity $(n>0)$, being a block-balanced identity are all derivation stable. Evidently, a property $\mathcal{P}$ of an identity is derivation-stable if and only if $\mathcal{P}$ defines a fully invariant congruence $\left(\sim_{S}\right)$ on the free semigroup corresponding to some semigroup $S$. Each row of Table 1 corresponds to a variety of monoids. Each variety in Table 1 is defined in three different ways: by the 
property of its identities, by its generating monoid and by its basis of identities. The first two rows of Table 1 correspond to the well-known varieties. The fact that the three descriptions in Rows 3-5 define the same variety is justified in Propositions 6.1, 4.2 and 6.2 respectively. The information in Rows 6-7 can be easily deduced from Theorem 3.1 in [7].

Proposition 6.1. For a monoid $S$ the following are equivalent:

(i) $E q(S)$ is the set of all block-balanced identities;

(ii) $S$ is finitely based by $\left\{\sigma_{1}, \sigma_{\mu}, \sigma_{2}\right\}^{\delta}$;

(iii) $S$ is equationally equivalent to $S\left(W_{A L}\right)$ where $W_{A L}$ is the set of all almostlinear words.

Proof. (i) $\leftrightarrow$ (ii) Notice that the identities $\sigma_{1}, \sigma_{\mu}$ and $\sigma_{2}$ are block-balanced. If an identity $\mathbf{u} \approx \mathbf{v}$ can be derived from $\left\{\sigma_{1}, \sigma_{\mu}, \sigma_{2}\right\}^{\delta}$, then in view of the fact that the property of being a block-balanced identity is derivation-stable, the identity $\mathbf{u} \approx \mathbf{v}$ is also block-balanced. The rest follows from Claim 1 in the proof of Theorem 3.3 .

(i) $\leftrightarrow$ (iii) First notice that $S\left(W_{A L}\right) \models\left\{\sigma_{1}, \sigma_{\mu}, \sigma_{2}\right\}$.

Let $\mathbf{u} \approx \mathbf{v}$ be an identity of $S\left(W_{A L}\right)$. If $\mathbf{u} \approx \mathbf{v}$ is not block-balanced, then for some $x \in \mathfrak{A}$, we have $\mathbf{u}(x, \operatorname{Lin}(\mathbf{u})) \neq \mathbf{v}(x, \operatorname{Lin}(\mathbf{u}))$. Since $S\left(W_{A L}\right)$ is a monoid, we have $S\left(W_{A L}\right) \models \mathbf{u}(x, \operatorname{Lin}(\mathbf{u})) \approx \mathbf{v}(x, \operatorname{Lin}(\mathbf{u}))$. But this is impossible because the word $\mathbf{u}(x, \operatorname{Lin}(\mathbf{u}))$ is an isoterm for $S\left(W_{A L}\right)$. Therefore, the monoid $S\left(W_{A L}\right)$ satisfies only block-balanced identities.

Proposition 6.2. For a monoid $S$ the following are equivalent:

(i) $E q(S)$ is the set of all block-balanced $\mathcal{P}_{1,2}$-identities;

(ii) $S$ is finitely based by $\left\{\sigma_{1}, \sigma_{2}\right\}^{\delta}$;

(iii) $S$ is equationally equivalent to $S\left(W_{A L} \cup\left\{a^{k} b^{k} \mid k>0\right\}\right)$.

Proof. (i) $\leftrightarrow$ (ii) First notice that $\sigma_{1}$ and $\sigma_{2}$ are block-balanced $\mathcal{P}_{1,2}$-identities. Since both properties are derivation-stable, any consequence of $\left\{\sigma_{1}, \sigma_{2}\right\}^{\delta}$ is again a blockbalanced $\mathcal{P}_{1,2}$-identity. The rest follows from Lemma 4.1.

(ii) $\leftrightarrow$ (iii) follows from Corollary 4.6.

Here are four more properties of identities similar to Property $\mathcal{P}_{1,2}$.

Definition 6.3. We say that an identity $\mathbf{u} \approx \mathbf{v}$ with $\operatorname{Lin}(\mathbf{u})=\operatorname{Lin}(\mathbf{v})$ and $\operatorname{Non}(\mathbf{u})=$ Non $(\mathbf{v})$ satisfies

(i) Property $\mathcal{P}_{1,1}$ if for each $x \neq y \in \operatorname{Cont}(\mathbf{u})$ we have $\left({ }_{1 \mathbf{u}} x\right)<_{\mathbf{u}}\left({ }_{1 \mathbf{u}} y\right)$ iff $\left({ }_{1 \mathbf{v}} x\right)<_{\mathbf{v}}$ $(1 \mathbf{v} y)$ (the order of first occurrences of variables is the same in $\mathbf{u}$ and in $\mathbf{v})$;

(ii) Property $\mathcal{P}_{2,2}$ if for each $x \neq y \in \operatorname{Cont}(\mathbf{u})$ we have $\left(_{\text {last } \mathbf{u}} x\right)<_{\mathbf{u}}\left(_{\text {last } \mathbf{u}} y\right)$ iff $\left(\right.$ last $\left.\mathbf{v}_{\mathbf{v}} x\right)<_{\mathbf{v}}($ last $\mathbf{v} y)$ (the order of last occurrences of variables is the same in $\mathbf{u}$ and in $\mathbf{v}$ );

(iii) Property $\mathcal{P}_{1,2}$ if for each $x \neq y \in \operatorname{Cont}(\mathbf{u})$ we have $\left({ }_{1 \mathbf{u}} x\right)<_{\mathbf{u}}($ lastu$y)$ iff $(1 \mathbf{v} x)<_{\mathbf{v}}($ last $y)$.

We say that a balanced identity $\mathbf{u} \approx \mathbf{v}$ satisfies 
(iv) Property $\mathcal{P}_{1, \mu}$ if for each $x \neq y \in \operatorname{Cont}(\mathbf{u})$ and each $1 \leq i \leq \operatorname{occ}_{\mathbf{u}}(y)$ we have $\left({ }_{1 \mathbf{u}} x\right)<_{\mathbf{u}}\left({ }_{i \mathbf{u}} y\right)$ iff $\left({ }_{1 \mathbf{v}} x\right)<_{\mathbf{v}}\left({ }_{i \mathbf{v}} y\right)$;

(v) Property $\mathcal{P}_{\mu, 2}$ if for each $x \neq y \in \operatorname{Cont}(\mathbf{u})$ and each $1 \leq i \leq \operatorname{occ}_{\mathbf{u}}(x)$ we have $\left({ }_{i \mathbf{u}} x\right)<_{\mathbf{u}}\left({ }_{\text {last } \mathbf{u}} y\right)$ iff $\left({ }_{i \mathbf{v}} x\right)<_{\mathbf{v}}\left({ }_{\text {last } \mathbf{v}} y\right)$.

The following machinery is needed only to prove Theorem 6.5.

We say that a set of identities $\Sigma$ is full if each identity $(\mathbf{u} \approx \mathbf{v}) \in \Sigma$ satisfies the following condition:

${ }^{*}$ ) If the words $\mathbf{u}$ and $\mathbf{v}$ do not begin (end) with the same linear letter, then $\Sigma$ contains the identity $t \mathbf{u} \approx t \mathbf{v}(\mathbf{u} t \approx \mathbf{v} t)$ for some $t \notin \operatorname{Cont}(\mathbf{u v})$

For example, if $\Sigma$ is a full set of identities containing $\sigma_{\mu}: x t_{1} x y t_{2} y \approx x t_{1} y x t_{2} y$, then $\Sigma$ must also contain the identities $t x t_{1} x y t_{2} y \approx t x t_{1} y x t_{2} y, x t_{1} x y t_{2} y t \approx x t_{1} y x t_{2} y t$ and $t x t_{1} x y t_{2} y t_{3} \approx t x t_{1} y x t_{2} y t_{3}$.

A substitution $\Theta: \mathfrak{A} \rightarrow \mathfrak{A}^{+}$is a homomorphism of the free semigroup $\mathfrak{A}^{+}$. Let $\Sigma$ be a full set of identities. A derivation of an identity $\mathbf{U} \approx \mathbf{V}$ from $\Sigma$ is a sequence of words $\mathbf{U}=\mathbf{U}_{1} \approx \mathbf{U}_{2} \approx \cdots \approx \mathbf{U}_{l}=\mathbf{V}$ and substitutions $\Theta_{1}, \ldots, \Theta_{l-1}\left(\mathfrak{A} \rightarrow \mathfrak{A}^{+}\right)$ so that for each $i=1, \ldots, l-1$ we have $\mathbf{U}_{i}=\Theta_{i}\left(\mathbf{u}_{i}\right)$ and $\mathbf{U}_{i+1}=\Theta_{i}\left(\mathbf{v}_{i}\right)$ for some identity $\mathbf{u}_{i} \approx \mathbf{v}_{i} \in \Sigma$. It is easy to see that each finite set of identities $\Sigma$ is a subset of a finite full set of identities $\Sigma^{\prime}$ so that $\operatorname{var} \Sigma=\operatorname{var} \Sigma^{\prime}$ and that an identity $\tau$ can be derived from $\Sigma$ in the usual sense if and only if $\tau$ can be derived from $\Sigma^{\prime}$ in the sense defined in the previous sentence.

We say that a property $\mathcal{P}$ of identities is substitution-stable provided that for every substitution $\Theta: \mathfrak{A} \rightarrow \mathfrak{A}^{+}$, the identity $\Theta(\mathbf{u}) \approx \Theta(\mathbf{v})$ satisfies property $\mathcal{P}$ whenever $\mathbf{u} \approx \mathbf{v}$ satisfies $\mathcal{P}$. Evidently, a property of identities is derivation-stable if and only if it is transitive and substitution-stable.

Let $\Theta: \mathfrak{A} \rightarrow \mathfrak{A}^{+}$be a substitution so that $\Theta(\mathbf{u})=\mathbf{U}$. Then $\Theta$ induces a map $\Theta_{\mathbf{u}}$ from $\operatorname{OccSet}(\mathbf{u})$ into subsets of $\operatorname{OccSet}(\mathbf{U})$ as follows. If $1 \leq i \leq \operatorname{occ}_{\mathbf{u}}(x)$ then $\Theta_{\mathbf{u}}\left({ }_{i \mathbf{u}} x\right)$ denotes the set of all elements of $\operatorname{OccSet}(\mathbf{U})$ contained in the subword of $\mathbf{U}$ of the form $\Theta(x)$ that corresponds to the $i^{\text {th }}$ occurrence of variable $x$ in $\mathbf{u}$. For example, if $\Theta(x)=a b$ and $\Theta(y)=b a b$ then $\Theta_{x y x}(2(x y x) x)=\left\{{ }_{3(a b b a b a b)} a,{ }_{4(a b b a b a b)} b\right\}$. Evidently, for each $x \in \operatorname{OccSet}(\mathbf{u})$ the set $\Theta_{\mathbf{u}}(x)$ is an interval in $\left(\operatorname{OccSet}(\mathbf{U}),<_{\mathbf{U}}\right)$. Now we define a function $\Theta_{\mathbf{u}}^{-1}$ from $\operatorname{OccSet}(\mathbf{U})$ to $\operatorname{OccSet}(\mathbf{u})$ as follows. If $c \in \operatorname{OccSet}(\mathbf{U})$ then $\Theta_{\mathbf{u}}^{-1}(c)=d$ so that $\Theta_{\mathbf{u}}(d)$ contains $c$. For example, $\Theta_{x y x}^{-1}(3($ abbabab $) a)={ }_{2(x y x)} x$. It is easy to see that if $\mathbf{U}=\Theta(\mathbf{u})$ then the function $\Theta_{\mathbf{u}}^{-1}$ is a homomorphism from $\left(\operatorname{OccSet}(\mathbf{U}),<_{\mathbf{U}}\right)$ to $\left(\operatorname{OccSet}(\mathbf{u}),<_{\mathbf{u}}\right)$, i.e. for every $c, d \in \operatorname{OccSet}(\mathbf{U})$ we have $\Theta_{\mathbf{u}}^{-1}(c) \leq_{\mathbf{u}} \Theta_{\mathbf{u}}^{-1}(d)$ whenever $c<\mathbf{u} d$.

If $X \subseteq \operatorname{OccSet}(\mathbf{u})$ and $f_{\mathbf{u}, \mathbf{v}}$ is an injection from a subset of OccSet(u) into the set $\operatorname{OccSet}(\mathbf{v})$ then we say that the set $X$ is $f_{\mathbf{u}, \mathbf{v}}$-stable in an identity $\mathbf{u} \approx \mathbf{v}$ if the map $f_{\mathbf{u}, \mathbf{v}}$ is defined on $X$ and is an isomorphism of the (totally) ordered sets $\left(X,<_{\mathbf{u}}\right)$ and $\left(f_{\mathbf{u}, \mathbf{v}}(X),<_{\mathbf{v}}\right)$. Otherwise, we say that the set $X$ is $f_{\mathbf{u}, \mathbf{v}}$-unstable in $\mathbf{u} \approx \mathbf{v}$. Let $e_{\mathbf{u}, \mathbf{v}}$ be a map from $\{1 \mathbf{u} x$, last $\mathbf{u} \mid x \in \operatorname{Non}(\mathbf{u}) \cap \operatorname{Non}(\mathbf{v})\}$ to $\{1 \mathbf{v} x$, last $\mathbf{v} \mid$ $x \in \operatorname{Non}(\mathbf{u}) \cap \operatorname{Non}(\mathbf{v})\}$ defined by $e_{\mathbf{u}, \mathbf{v}}\left({ }_{1 \mathbf{u}} x\right)={ }_{1 \mathbf{v}} x$ and $e_{\mathbf{u}, \mathbf{v}}($ last $\mathbf{u} x)=$ last $\mathbf{v} x$. The following lemma is needed only to prove Theorem 6.5. 
Lemma 6.4. Let $\mathbf{u} \approx \mathbf{v}$ be a $\mathcal{P}_{1,1}$-identity and $\Theta: \mathfrak{A} \rightarrow \mathfrak{A}^{+}$be a substitution. If $\mathbf{U}=\Theta(\mathbf{u})$ and $\mathbf{V}=\Theta(\mathbf{v})$ then for each $x \in \operatorname{Cont}(\mathbf{U})$ we have $\Theta_{\mathbf{u}}^{-1}\left({ }_{1} \mathbf{U} x\right)={ }_{1 \mathbf{u}} z$ and $\Theta_{\mathbf{v}}^{-1}(1 \mathbf{v} x)={ }_{1 \mathbf{v}} z$ for some $z \in \operatorname{Cont}(\mathbf{u})$.

Proof. Evidently, $\Theta_{\mathbf{u}}^{-1}\left({ }_{1} \mathbf{u} x\right)={ }_{1 \mathbf{u}} z$ and $\Theta_{\mathbf{v}}^{-1}\left({ }_{1 \mathbf{v}} x\right)={ }_{1 \mathbf{v}} y$ for some $z, y \in \operatorname{Cont}(\mathbf{u})$. If $z \neq y$ then both $\Theta(z)$ and $\Theta(y)$ contain $x$. Therefore, ${ }_{1 \mathbf{u}} z<_{\mathbf{u} 1 \mathbf{u}} y$ and ${ }_{1 \mathbf{v}} y<_{\mathbf{v}} 1 \mathbf{v} z$. To avoid a contradiction to the fact that the set $\left\{{ }_{1 \mathbf{u}} z,{ }_{1 \mathbf{u}} y\right\} \subseteq \operatorname{OccSet}(\mathbf{u})$ is $e_{\mathbf{u}, \mathbf{v}}$-stable in $\mathbf{u} \approx \mathbf{v}$, we must assume that $y=z$.

Theorem 6.5. All properties of identities in Definition 6.3 are derivation-stable.

Proof. Property $\mathcal{P}_{1,2}$ is derivation-stable by Proposition 4.2 .

(i) Let $\mathbf{u} \approx \mathbf{v}$ be a $\mathcal{P}_{1,1}$-identity and $\Theta: \mathfrak{A} \rightarrow \mathfrak{A}^{+}$be a substitution. Denote $\mathbf{U}=\Theta(\mathbf{u})$ and $\mathbf{V}=\Theta(\mathbf{v})$. Suppose that for some $x, y \in \operatorname{Cont}(\mathbf{U})$ we have ${ }_{1 \mathbf{U}} x<\mathbf{U}$ $1 \mathbf{u} y$. Then by Lemma 6.4 we have $\Theta_{\mathbf{u}}^{-1}\left({ }_{1} \mathbf{u} x\right)={ }_{1 \mathbf{u}} z, \Theta_{\mathbf{v}}^{-1}(1 \mathbf{v} x)={ }_{1 \mathbf{v}} z$ for some $z \in \operatorname{Cont}(\mathbf{u}), \Theta_{\mathbf{u}}^{-1}\left({ }_{1} \mathbf{u} y\right)={ }_{1 \mathbf{u}} p$ and $\Theta_{\mathbf{v}}^{-1}\left({ }_{1} \mathbf{v} y\right)={ }_{1 \mathbf{v}} p$ for some $p \in \operatorname{Cont}(\mathbf{u})$.

Since $\Theta_{\mathbf{u}}^{-1}$ is a homomorphism from $\left(\operatorname{OccSet}(\mathbf{U}),<_{\mathbf{U}}\right)$ to $\left(\operatorname{OccSet}(\mathbf{u}),<_{\mathbf{u}}\right)$, we have that ${ }_{1 \mathbf{u}} z \leq_{\mathbf{u} 1 \mathbf{u}} p$. Since the identity $\mathbf{u} \approx \mathbf{v}$ satisfies Property $\mathcal{P}_{1,1}$, we have ${ }_{1 \mathbf{v}} z \leq_{\mathbf{v} 1 \mathbf{v}} p$. If $z \neq p$ then we have ${ }_{1 \mathbf{v}} x<_{\mathbf{v} 1 \mathbf{v}} y$ because the map $l_{\mathbf{U}, \mathbf{v}}$ restricted to the set $\{1 \mathbf{U} x, 1 \mathbf{U} y\}$ is a composition of three isomorphisms: $\Theta_{\mathbf{u}}^{-1} \circ e_{\mathbf{u}, \mathbf{v}} \circ\left(\Theta_{\mathbf{v}}^{-1}\right)^{-1}$.

If $z=p$ then using the fact that the ordered sets $\left(\Theta_{\mathbf{u}}(1 \mathbf{u} z),<_{\mathbf{U}}\right)$ and $\left(\Theta_{\mathbf{v}}(1 \mathbf{v} z),<_{\mathbf{v}}\right.$ ) correspond to the same word $\Theta(z)$, it is easy to show that ${ }_{1 \mathbf{v}} x<_{\mathbf{v}} 1 \mathbf{v} y$. In either case, the pair $\left\{1 \mathbf{U} x,{ }_{1} \mathbf{U} y\right\}$ is $e_{\mathbf{u}, \mathbf{v}}$-stable in $\mathbf{U} \approx \mathbf{V}$. Therefore, the identity $\mathbf{U} \approx \mathbf{V}$ also satisfies Property $\mathcal{P}_{1,1}$. Thus, we have proved that Property $\mathcal{P}_{1,1}$ is substitutionstable.

(ii) Let $\mathbf{u} \approx \mathbf{v}$ be a $\mathcal{P}_{1, \mu}$-identity and $\Theta: \mathfrak{A} \rightarrow \mathfrak{A}^{+}$be a substitution. Denote $\mathbf{U}=\Theta(\mathbf{u})$ and $\mathbf{V}=\Theta(\mathbf{v})$. Let $x \neq y \in \operatorname{Cont}(\mathbf{U})$. Since Property $\mathcal{P}_{1, \mu}$ is stronger than $\mathcal{P}_{1,1}$, by Lemma 6.4 we may assume that $\Theta_{\mathbf{u}}^{-1}\left({ }_{1 \mathbf{U}} x\right)={ }_{1 \mathbf{u}} x$ and $\Theta_{\mathbf{v}}^{-1}\left({ }_{1} \mathbf{v} x\right)=$ ${ }_{1 \mathbf{v}} x$. Since $\mathbf{u} \approx \mathbf{v}$ is a balanced identity we identify $\operatorname{OccSet}(\mathbf{u})$ and $\operatorname{OccSet}(\mathbf{v})$. In particular, we identify ${ }_{1 \mathbf{u}} x$ and ${ }_{1 \mathbf{v}} x$.

Define $\left.\Theta_{\mathbf{u}}^{-1}(y):=\left\{c \in \operatorname{OccSet}(\mathbf{u}) \mid c=\Theta_{\mathbf{u}}^{-1}{ }_{(i \mathbf{U}} y\right), 1 \leq i \leq \operatorname{occ}_{\mathbf{U}}(y)\right\}$. Define $Y_{\mathbf{u}}:=\left\{c \in \Theta_{\mathbf{u}}^{-1}(y) \mid c \leq_{\mathbf{u}}\left({ }_{1 \mathbf{u}} x\right)\right\}$. Since $\mathbf{u} \approx \mathbf{v}$ satisfies Property $\mathcal{P}_{1, \mu}$, we have $Y_{\mathbf{u}}=Y_{\mathbf{v}}$. This implies that the number of occurrences of $y$ which precede ${ }_{1} \mathbf{U} x$ in $\mathbf{U}$ is the same as the number of occurrences of $y$ which precede ${ }_{1} \mathbf{v} x$ in $\mathbf{V}$. Therefore, the identity $\mathbf{U} \approx \mathbf{V}$ also satisfies Property $\mathcal{P}_{1, \mu}$. Thus, we have proved that Property $\mathcal{P}_{1, \mu}$ is substitution-stable.

Properties $\mathcal{P}_{2,2}$ and $\mathcal{P}_{\mu, 2}$ are substitution-stable by dual arguments. Since all properties of identities in Definition 6.3 are transitive (obvious) and substitutionstable, all these properties are derivation-stable.

With each subset $\Sigma$ of $\left\{\sigma_{1}, \sigma_{\mu}, \sigma_{2}\right\}$ we associate an assignment of two Types to all pairs of occurrences of distinct non-linear variables in all words as follows. We say that each pair of occurrences of two distinct non-linear variables in each word is $\left\{\sigma_{1}, \sigma_{\mu}, \sigma_{2}\right\}$-good. If $\Sigma$ is a proper subset of $\left\{\sigma_{1}, \sigma_{\mu}, \sigma_{2}\right\}$, then we say that a pair of occurrences of distinct non-linear variables is $\Sigma$-good if it is not declared to be $\Sigma$-bad in the following definition. 
Definition 6.6. If $\{c, d\} \subseteq \operatorname{OccSet}(\mathbf{u})$ is a pair of occurrences of two distinct nonlinear variables $x \neq y$ in a word $\mathbf{u}$ then

(i) pair $\{c, d\}$ is $\left\{\sigma_{\mu}, \sigma_{2}\right\}$-bad if $\{c, d\}=\left\{{ }_{1 \mathbf{u}} x,{ }_{1 \mathbf{u}} y\right\}$;

(ii) pair $\{c, d\}$ is $\left\{\sigma_{1}, \sigma_{\mu}\right\}$-bad if $\{c, d\}=\{$ lastu $x$, last $\mathbf{u} y\}$;

(iii) pair $\{c, d\}$ is $\left\{\sigma_{1}, \sigma_{2}\right\}$-bad if $\{c, d\}=\{1 \mathbf{u} x$, lastu $y\}$.

(iv) pair $\{c, d\}$ is $\sigma_{\mu}$-bad if $\{c, d\}=\left\{{ }_{1 \mathbf{u}} x,{ }_{1 \mathbf{u}} y\right\}$ or $\{c, d\}=\left\{\right.$ lastu $_{\mathbf{u}} x$, last $\left.\mathbf{u} y\right\}$;

(v) pair $\{c, d\}$ is $\sigma_{2}$-bad if $c={ }_{1 \mathbf{u}} x$ or $d={ }_{1 \mathbf{u}} y$;

(vi) pair $\{c, d\}$ is $\sigma_{1}$-bad if $c=\ell \mathbf{u} x$ or $d=\ell \mathbf{u} y$.

The following theorem describes the equational theories for each of the seven varieties defined by the seven subsets of $\left\{\sigma_{1}, \sigma_{\mu}, \sigma_{2}\right\}$. In particular, it generalizes both Proposition 6.1((i) $\leftrightarrow$ (ii)) and Proposition 6.2((i) $\leftrightarrow$ (ii)).

Theorem 6.7. If $\Sigma \subseteq\left\{\sigma_{1}, \sigma_{\mu}, \sigma_{2}\right\}$ then for every identity $\mathbf{u} \approx \mathbf{v}$ the following conditions are equivalent:

(i) $\mathbf{u} \approx \mathbf{v}$ is block-balanced and each $\Sigma$-bad pair of occurrences of two distinct non-linear variables in $\mathbf{u}$ is stable in $\mathbf{u} \approx \mathbf{v}$;

(ii) $\mathbf{u} \approx \mathbf{v}$ can be derived from $\Sigma^{\delta}$ by swapping $\Sigma$-good adjacent pairs of occurrences;

(iii) $\mathbf{u} \approx \mathbf{v}$ is satisfied by $\operatorname{var}\left(\Sigma^{\delta}\right)$.

Proof. (i) $\rightarrow$ (ii) We assign a Type to each pair $\{c, d\} \subseteq$ OccSet $(\mathbf{u})$ of occurrences of distinct non-linear variables in a word $\mathbf{u}$ as follows. If $\{c, d\}$ is $\Sigma$-good then we say that $\{c, d\}$ is of Type 1 . Otherwise, $\{c, d\}$ is of Type 2 .

Let $\mathbf{u} \approx \mathbf{v}$ be a block-balanced identity so that each $\Sigma$-bad pair of occurrences of two distinct non-linear variables in $\mathbf{u}$ is stable in $\mathbf{u} \approx \mathbf{v}$. Let $\{c, d\} \subseteq \operatorname{OccSet}(\mathbf{u})$ be a critical pair in $\mathbf{u} \approx \mathbf{v}$. Suppose that $\{c, d\}$ is of Type 1 . Then using an identity from $\Sigma^{\delta}$ and swapping $c$ and $d$ in $\mathbf{u}$ we obtain some word $\mathbf{w}$. Evidently, the word $\mathbf{w}$ satisfies all the requirements of Lemma 3.4. Notice that the identity $\mathbf{u} \approx \mathbf{v}$ does not have any unstable pairs of Type 2.

(ii) $\rightarrow$ (iii) Obvious.

(iii) $\rightarrow$ (i) Notice that each identity in $(\mathbf{u} \approx \mathbf{v}) \in \Sigma^{\delta}$ is block-balanced and each $\Sigma$-bad pair of occurrences of two distinct non-linear variables in $\mathbf{u}$ is stable in $\mathbf{u} \approx \mathbf{v}$. By Theorem 6.5 this property is derivation-stable.

Here are notation-free reformulations of some statements contained in Theorem 6.7

Corollary 6.8. (i) An identity is a consequence of $\left\{\sigma_{\mu}\right\}^{\delta}$ if and only if it is blockbalanced and the orders of the first and the last occurrences of variables in its left and right sides are the same;

(ii) An identity is a consequence of $\left\{\sigma_{1}, \sigma_{\mu}\right\}^{\delta}$ if and only it is block-balanced and the order of the last occurrences of variables in its left and right sides is the same;

(iii) An identity is a consequence of $\left\{\sigma_{2}, \sigma_{\mu}\right\}^{\delta}$ if and only if it is block-balanced and the order of the first occurrences of variables in its left and right sides is the same. 


\section{Another proof that the monoid of all reflexive binary relations on a four-element set is finitely based}

Recall from section 2 that by the result of Volkov [18], $J_{3}$ is the equational theory of the monoid of all reflexive binary relations on a four-element set. In this section we use Lemma 3.1 to reprove the following result of Blanchet-Sadri.

Theorem 7.1. [2, Theorem 3.6] The set of identities $J_{3}$ is finitely based by $\left\{x t_{1} x t_{2} x \approx\right.$ $\left.x t_{1} x x t_{2} x, x t_{1} y x t_{2} y \approx x t_{1} x y x t_{2} y, y t_{1} x x y t_{2} x \approx y t_{1} x x y x t_{2} x,(x y)^{3} \approx(y x)^{3}\right\}$.

It is easy to check that $J_{3}$ contains the following sets of identities:

$\Sigma=\{$ xyxytxty $\approx$ yxyxtxty, xtytxyxy $\approx$ xtytyxy $\}$;

$\Delta=\{$ xtytxytxty $\approx$ xtytyxtxty, xtytxytytx $\approx$ xtytyxtyt $x\}$.

One can verify that in the presence of

$\left\{x t_{1} x t_{2} x \approx x t_{1} x x t_{2} x, x t_{1} y x t_{2} y \approx x t_{1} x y x x t_{2} y, y t_{1} x x y t_{2} x \approx y t_{1} x x y t_{2} x\right\}$ the identity $(x y)^{3} \approx(y x)^{3}$ is equivalent to $\Sigma \cup \Delta$. The next theorem claims a larger basis for $J_{3}$ than Theorem 7.1 but the identities in this basis still contain only two non-linear variables.

Theorem 7.2. The set of identities $J_{3}$ is finitely based by $\left\{x t_{1} x t_{2} x \approx x t_{1} x x t_{2} x, x t_{1} y x x t_{2} y \approx\right.$ $\left.x t_{1} x y x t_{2} y, y t_{1} x x y t_{2} x \approx y t_{1} x x y t_{2} x\right\} \cup \Sigma \cup \Delta$.

Theorem 7.2 is an immediate consequence of Lemmas 7.4 and 7.5. The length of a word $\mathbf{u}$ is the cardinality of $\operatorname{OccSet}(\mathbf{u})$. First, we need one auxiliary lemma.

Lemma 7.3. $\left\{x t_{1} x t_{2} x \approx x t_{1} x x t_{2} x, x t_{1} y x x t_{2} y \approx x t_{1} x y x x t_{2} y\right\}^{\delta} \vdash \mathbf{A B} x \mathbf{C} \approx \mathbf{A} x \mathbf{B} x \mathbf{C}$ whenever $x \in \operatorname{Cont}(\mathbf{A}), x \in \operatorname{Cont}(\mathbf{C})$ and $\operatorname{Cont}(\mathbf{B}) \subseteq \operatorname{Cont}(\mathbf{C})$.

Proof. Evidently, $\left\{x t_{1} x t_{2} x \approx x t_{1} x x t_{2} x\right\}^{\delta} \vdash \mathbf{A B} x \mathbf{C} \approx \mathbf{A B} x x \mathbf{C}$.

If $\mathbf{u}=\mathbf{A B} x x \mathbf{C}$ then $\operatorname{OccSet}(\mathbf{u})=a_{1} \ll_{\mathbf{u}} a_{2} \ll_{\mathbf{u}} \cdots \ll_{\mathbf{u}} a_{k} \ll_{\mathbf{u}} b_{1} \ll_{\mathbf{u}} b_{2} \ll_{\mathbf{u}}$ $\cdots \ll_{\mathbf{u}} b_{p} \ll_{\mathbf{u}}\left({ }_{i \mathbf{u}} x\right) \ll_{\mathbf{u}}\left({ }_{(i+1) \mathbf{u}} x\right) \ll_{\mathbf{u}} c_{1} \ll_{\mathbf{u}} c_{2} \ll_{\mathbf{u}} \cdots \ll_{\mathbf{u}} c_{q}$, where $k, p, q \geq 0$ are the lengths of $\mathbf{A}, \mathbf{B}, \mathbf{C}$ respectively and $1<i<i+1<\operatorname{occ}_{\mathbf{u}}(x)$.

By our assumption, for each $1 \leq i \leq p, b_{i} \in \operatorname{OccSet}(\mathbf{u})$ is not the last occurrence in $\mathbf{u}$ of some variable $y \in \operatorname{Cont}(\mathbf{B})$. Therefore, $\left\{x t_{1} y x x t_{2} y \approx x t_{1} x y x x t_{2} y\right\}^{\delta} \vdash$ $\mathbf{A B} x x \mathbf{C} \approx \mathbf{u}_{\mathbf{1}}$, where $\operatorname{OccSet}\left(\mathbf{u}_{\mathbf{1}}\right)=a_{1} \ll_{\mathbf{u}} a_{2} \ll_{\mathbf{u}} \cdots \ll_{\mathbf{u}} a_{k} \ll_{\mathbf{u}} b_{1} \ll_{\mathbf{u}} b_{2} \ll_{\mathbf{u}}$ $\cdots \ll_{\mathbf{u}}\left({ }_{i \mathbf{u}} x\right) \ll_{\mathbf{u}} b_{p} \ll_{\mathbf{u}}\left({ }_{(i+1) \mathbf{u}} x\right)\left({ }_{(i+2) \mathbf{u}} x\right) \ll_{\mathbf{u}} c_{1} \ll_{\mathbf{u}} c_{2} \ll_{\mathbf{u}} \cdots \ll_{\mathbf{u}} c_{q}$.

And so on. After applying the identities in $\left\{x t_{1} x t_{2} x \approx x t_{1} x x t_{2} x, x t_{1} y x t_{2} y \approx\right.$ $\left.x t_{1} x y x t_{2} y\right\}^{\delta} p$ times, we obtain $\mathbf{u}_{\mathbf{p}}=\mathbf{A} x \mathbf{B} x \mathbf{C}$.

We will use the properties of identities in Definition 6.3. For each $\mathcal{P}_{1}$-identity $\mathbf{u} \approx \mathbf{v}$ we define

- $\operatorname{Dist}\left(\mathcal{P}_{1} \rightarrow \mathcal{P}_{1,1} \wedge \mathcal{P}_{2,2}\right)(\mathbf{u} \approx \mathbf{v}):=\left\{\left\{1 \mathbf{u} x,{ }_{1 \mathbf{u}} y\right\} \mid x, y \in \operatorname{Cont}(\mathbf{u}),{ }_{1 \mathbf{u}} x<_{\mathbf{u}}\right.$ $\left.{ }_{1 \mathbf{u}} y,{ }_{\mathbf{v}} y<_{\mathbf{v}} 1 \mathbf{v} x\right\} \cup\{\{$ last $\mathbf{u} x$, last $\mathbf{u}\}\} \mid x, y \in \operatorname{Cont}(\mathbf{u})$, last $\mathbf{u} x<_{\mathbf{u}}$ last $\mathbf{u} y$, last $\mathbf{v} y<_{\mathbf{v}}$ last $\left.\mathbf{v} x\right\}$.

In other words, $\operatorname{Dist}\left(\mathcal{P}_{1} \rightarrow \mathcal{P}_{1,1} \wedge \mathcal{P}_{2,2}\right)(\mathbf{u} \approx \mathbf{v})$ is the set of all unstable pairs of the form $\left\{{ }_{1 \mathbf{u}} x,{ }_{1 \mathbf{u}} y\right\}$ or $\{$ last $\mathbf{u} x$, last $\mathbf{u}\}$ in a $\mathcal{P}_{1}$-identity $\mathbf{u} \approx \mathbf{v}$. It is easy to see 
that the set $\operatorname{Dist}\left(\mathcal{P}_{1} \rightarrow \mathcal{P}_{1,1} \wedge \mathcal{P}_{2,2}\right)(\mathbf{u} \approx \mathbf{v})$ is empty if and only if $\mathbf{u} \approx \mathbf{v}$ is a $\mathcal{P}_{1,1} \wedge \mathcal{P}_{2,2}$-identity.

Lemma 7.4. Every identity in $J_{3}$ can be derived from $\left\{x t_{1} x t_{2} x \approx x t_{1} x x t_{2} x, x t_{1} y x t_{2} y \approx\right.$ $\left.x t_{1} x y x t_{2} y, y t_{1} x x y t_{2} x \approx y t_{1} x x y x t_{2} x\right\}^{\delta} \cup \Sigma^{\delta}$ and from a $\mathcal{P}_{1,1} \wedge \mathcal{P}_{1,2} \wedge \mathcal{P}_{2,2}$-identity in $J_{3}$.

Proof. Let $\mathbf{u} \approx \mathbf{v}$ be an identity in $J_{3}$. Since $J_{3} \subset J_{2}$, Proposition 4.2 implies that every identity in $J_{3}$ has Property $\mathcal{P}_{1,2}$. Suppose that $\mathbf{u} \approx \mathbf{v}$ does not have Property $\mathcal{P}_{1,1}$. Then for some $x \neq y \in \operatorname{Cont}(\mathbf{u})$ we have that ${ }_{1 \mathbf{u}} x<_{\mathbf{u}} 1 \mathbf{u} y,{ }_{1 \mathbf{v}} y<_{\mathbf{v}} 1 \mathbf{v} x$ and for each $c \in \operatorname{OccSet}(\mathbf{u})$ such that ${ }_{1 \mathbf{u}} x<_{\mathbf{u}} c<_{\mathbf{u}} 1 \mathbf{u} y, c$ is neither the first nor the last occurrence of some variable $z$ with $\operatorname{occ}_{\mathbf{u}}(z) \geq 3$.

Claim 11. $\operatorname{occ}_{\mathbf{u}}(x) \geq 3$ and $\operatorname{occ}_{\mathbf{u}}(y) \geq 3$.

Proof. First, suppose that one of the variables, say $y$, is linear. Then the word $\mathbf{u}$ contains the scattered subword $x y$ but the word $\mathbf{v}$ does not contain the scattered subword $x y$. If $o_{c} c_{\mathbf{u}}(y)=2$ then the word $\mathbf{u}$ contains the scattered subword $x y y$ but the word $\mathbf{v}$ does not contain the scattered subword $x y y$. To avoid a contradiction, we must assume that $\operatorname{occ}_{\mathbf{u}}(x) \geq 3$ and $\operatorname{occ}_{\mathbf{u}}(y) \geq 3$.

Let $d \in \operatorname{OccSet}(\mathbf{u})$ be minimal in order $<_{\mathbf{u}}$ such that ${ }_{1 \mathbf{u}} y<_{\mathbf{u}} d$ and $d$ is the last occurrence of some variable $p \in \operatorname{Cont}(\mathbf{u})$. (The variable $p$ may coincide with $x$ or $y)$.

Claim 12. If the word $\mathbf{u}$ contains an occurrence of a variable $z$ between $1 \mathbf{u} x$ and ${ }_{1 \mathbf{u}} y$ then the word $\mathbf{u}$ also contains an occurrence of $z$ between ${ }_{1 \mathbf{u}} y$ and lastu $p$. (The variable z may coincide with $x$ or $p$ ).

Proof. Since $\mathbf{u} \approx \mathbf{v}$ is a $\mathcal{P}_{1,2}$-identity, we have that ${ }_{1 \mathbf{v}} y<_{\mathbf{v}}{ }_{1 \mathbf{v}} x<_{\mathbf{v}}$ last $\mathbf{v}$.

To obtain a contradiction, assume that the variable $z$ does not appear between ${ }_{\mathbf{u}} y$ and ${ }_{l a s t \mathbf{u}} p$. Then the word $\mathbf{u}$ does not contain the scattered subword $y z p$. Consequently, there is no occurrence of $z$ between ${ }_{1 \mathbf{v}} y$ and ${ }_{\text {last }} p$ neither. Therefore, the word $\mathbf{v}$ does not contain the scattered subword $x z p$; a contradiction.

Using Lemma 7.3 we erase all occurrences of variables (if any) between ${ }_{1 \mathbf{u}} x$ and ${ }_{1 \mathbf{u}} y$ and denote the resulting word by $\mathbf{w}_{\mathbf{1}}$. Notice that $\left({ }_{1 \mathbf{w}_{1}} x\right) \ll_{\mathbf{w}_{\mathbf{1}}}\left({ }_{1 \mathbf{w}_{1}} y\right)$. Lemma 7.3 implies that $\left\{x t_{1} x t_{2} x \approx x t_{1} x x t_{2} x, x t_{1} y x x t_{2} y \approx x t_{1} x y x x t_{2} y\right\}^{\delta} \vdash \mathbf{u} \approx \mathbf{w}_{\mathbf{1}}$.

Claim 13. If for some $c \in \operatorname{OccSet}\left(\mathbf{w}_{\mathbf{1}}\right)$ we have $\left({ }_{1 \mathbf{w}_{\mathbf{1}}} y\right)<_{\mathbf{w}_{\mathbf{1}}} c<_{\mathbf{w}_{\mathbf{1}}}\left({ }_{2 \mathbf{w}_{\mathbf{1}}} x\right)$ then $c$ is not the last occurrence of some variable $z \neq x$ with occ $_{\mathrm{w}_{1}}(z) \geq 2$;

Proof. Suppose that $c={ }_{\text {last } \mathbf{w}_{\mathbf{1}}} z$ for some $z \in \operatorname{Cont}\left(\mathbf{w}_{\mathbf{1}}\right)$. (This includes the case when $z$ is linear in $\mathbf{w}_{\mathbf{1}}$.)

Since the word $\mathbf{w}_{\mathbf{1}}$ contains the scattered subword $x z x$, the word $\mathbf{v}$ also contains the scattered subword $x z x$. Therefore, we must have ${ }_{1 \mathbf{v}} y<_{\mathbf{v} 1 \mathbf{v}} x<_{\mathbf{v}}$ last $z$. Now the word $\mathbf{v}$ contains the scattered subword $y x z$. So, the word $\mathbf{w}_{\mathbf{1}}$ must also contain the scattered subword $y x z$. This contradicts the fact that $c={ }_{\text {last } \mathbf{w}_{1}} z$. 
Using Lemma 7.3 we insert an occurrence of $x$ in $\mathbf{w}_{\mathbf{1}}$ right after ${ }_{1 \mathbf{w}_{\mathbf{1}}} y$ and denote the resulting word by $\mathbf{w}_{\mathbf{2}}$. Notice that $\left({ }_{1 \mathbf{w}_{\mathbf{2}}} x\right) \ll_{\mathbf{w}_{\mathbf{2}}}\left({ }_{1 \mathbf{w}_{\mathbf{2}}} y\right) \ll_{\mathbf{w}_{\mathbf{2}}}\left({ }_{2 \mathbf{w}_{\mathbf{2}}} x\right)$. Lemma 7.3 implies that $\left\{x t_{1} x t_{2} x \approx x t_{1} x x t_{2} x, x t_{1} y x x t_{2} y \approx x t_{1} x y x x t_{2} y\right\}^{\delta} \vdash \mathbf{w}_{\mathbf{1}} \approx \mathbf{w}_{\mathbf{2}}$.

Claim 14. If for some $c \in \operatorname{OccSet}\left(\mathbf{w}_{\mathbf{2}}\right)$ we have $\left({ }_{2 \mathbf{w}_{\mathbf{2}}} x\right)<_{\mathbf{w}_{\mathbf{2}}} c<_{\mathbf{w}_{\mathbf{2}}}\left({ }_{2 \mathbf{w}_{\mathbf{2}}} y\right)$ then $c$ is not the last occurrence of some variable $z \neq y$ with occ $_{\mathrm{w}_{2}}(z) \geq 2$;

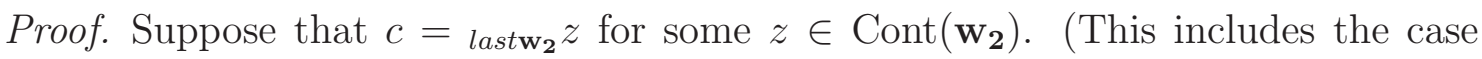
when $z$ is linear in $\mathbf{w}_{\mathbf{2}}$.)

Since the word $\mathbf{w}_{\mathbf{2}}$ contains the scattered subword $x y z$, the word $\mathbf{v}$ also contains the scattered subword $x y z$. Therefore, we must have ${ }_{1 \mathbf{v}} y<_{\mathbf{v} 1 \mathbf{v}} x<_{\mathbf{v}} 2 \mathbf{v} y<_{\mathbf{v}}$ last $z$. Now the word $\mathbf{v}$ contains the scattered subword yyz. So, the word $\mathbf{u}$ must also contain the scattered subword $y y z$. This contradicts the fact that $c={ }_{l_{\text {ast }} \mathbf{w}_{2}} z$.

Using Lemma 7.3 we insert an occurrence of $y$ in $\mathbf{w}_{\mathbf{2}}$ right after ${ }_{2 \mathbf{w}_{2}} x$ and denote the resulting word by $\mathbf{w}_{\mathbf{3}}$. Notice that $\left({ }_{1 \mathbf{w}_{\mathbf{3}}} x\right) \ll_{\mathbf{w}_{\mathbf{3}}}\left(1 \mathbf{w}_{\mathbf{3}} y\right) \ll_{\mathbf{w}_{\mathbf{3}}}\left({ }_{2 \mathbf{w}_{\mathbf{3}}} x\right) \ll_{\mathbf{w}_{\mathbf{3}}}\left(2 \mathbf{w}_{\mathbf{3}} y\right)$. Lemma 7.3 implies that $\left\{x t_{1} x t_{2} x \approx x t_{1} x x t_{2} x, x t_{1} y x x t_{2} y \approx x t_{1} x y x x t_{2} y\right\}^{\delta} \vdash \mathbf{w}_{2} \approx$ $\mathbf{w}_{3}$.

Now we apply an identity from $\Sigma^{\delta}$ to $\mathbf{w}_{\mathbf{3}}$ and obtain a word $\mathbf{u}_{\mathbf{1}}$. Notice that $\left|\operatorname{Dist}\left(\mathcal{P}_{1,2} \rightarrow \mathcal{P}_{1,1} \wedge \mathcal{P}_{1,2} \wedge \mathcal{P}_{2,2}\right)\left(\mathbf{u}_{\mathbf{1}} \approx \mathbf{v}\right)\right|<\left|\operatorname{Dist}\left(\mathcal{P}_{1,2} \rightarrow \mathcal{P}_{1,1} \wedge \mathcal{P}_{1,2} \wedge \mathcal{P}_{2,2}\right)(\mathbf{u} \approx \mathbf{v})\right|$. If $\mathbf{u} \approx \mathbf{v}$ does not have Property $\mathcal{P}_{2,2}$ we use the dual arguments and the dual of Lemma 7.3 (in particular, we use the dual identity $y t_{1} x x y t_{2} x \approx y t_{1} x x y x t_{2} x$ ).

Lemma 3.1 implies that every identity of $S$ can be derived from $\left\{x t_{1} x t_{2} x \approx\right.$ $\left.x t_{1} x x t_{2} x, x t_{1} y x x t_{2} y \approx x t_{1} x y x t_{2} y, y t_{1} x x y t_{2} x \approx y t_{1} x x y x t_{2} x\right\}^{\delta} \cup \Sigma^{\delta}$ and from a $\mathcal{P}_{1,1} \wedge$ $\mathcal{P}_{1,2} \wedge \mathcal{P}_{2,2}$-identity in $J_{3}$.

We say that a 12-block in $\mathbf{u}$ is a maximal subword of $\mathbf{u}$ which contains neither first nor last occurrences of variables. Evidently, a 12-block in $\mathbf{u}$ may contain only occurrences of variables $x$ with occ $_{\mathbf{u}}(x) \geq 3$. If $\mathbf{u} \approx \mathbf{v}$ is a $\left(\mathcal{P}_{1,1} \wedge \mathcal{P}_{2,2} \wedge \mathcal{P}_{1,2}\right)$-identity, then the sequences of the first and the last occurrences of variables in $\mathbf{u}$ and $\mathbf{v}$ are the same. If $\mathbf{B}$ is a 12-block in $\mathbf{u}$, then the corresponding 12-block $\mathbf{B}^{\prime}$ in $\mathbf{v}$ is located between the corresponding first and last occurrences of variables.

Lemma 7.5. Every $\left(\mathcal{P}_{1,1} \wedge \mathcal{P}_{2,2} \wedge \mathcal{P}_{1,2}\right)$-identity in $J_{3}$ can be derived from $\left\{x t_{1} x t_{2} x \approx\right.$ $\left.x t_{1} x x t_{2} x, x t_{1} y x t_{2} y \approx x t_{1} x y x t_{2} y, y t_{1} x x y t_{2} x \approx y t_{1} x x y x t_{2} x\right\}^{\delta} \cup \Delta^{\delta}$.

Proof. Let $\mathbf{u} \approx \mathbf{v}$ be a $\left(\mathcal{P}_{1,1} \wedge \mathcal{P}_{2,2} \wedge \mathcal{P}_{1,2}\right)$-identity in $J_{3}$.

Claim 15. Suppose that a 12-block $\mathbf{B}$ in $\mathbf{u}$ contains an occurrence of $z \in \operatorname{Cont}(\mathbf{u})$ but the corresponding block $\mathbf{B}^{\prime}$ in $\mathbf{v}$ contains no occurrences of $z$. Then $\left\{x t_{1} x t_{2} x \approx\right.$ $\left.x t_{1} x x t_{2} x, x t_{1} y x t_{2} y \approx x t_{1} x y x t_{2} y, y t_{1} x x y t_{2} x \approx y t_{1} x x y x t_{2} x\right\}^{\delta} \vdash \mathbf{v} \approx \mathbf{w}$ such that the corresponding block $\mathbf{B}^{\prime \prime}$ in $\mathbf{w}$ contains an occurrence of $z$.

Proof. Let $c \in \operatorname{OccSet}(\mathbf{u})$ denote the occurrence of $z$ in B. Let $d_{1} \in \operatorname{OccSet}(\mathbf{u})$ be maximal in order $<_{\mathbf{u}}$ such that $d_{1}<_{\mathbf{u}} c$ and $d_{1}$ is the first occurrence of some variable $q \in \operatorname{Cont}(\mathbf{u})$. (The variable $q$ may coincide with $z$.) Let $d_{2} \in \operatorname{OccSet}(\mathbf{u})$ be 
minimal in order $<_{\mathbf{u}}$ such that $c<_{\mathbf{u}} d_{2}$ and $d_{2}$ is the last occurrence of some variable $p \in \operatorname{Cont}(\mathbf{u})$. (The variable $p$ may coincide with $z$.)

Since the word $\mathbf{u}$ contains the scattered subword $q z p$, the word $\mathbf{v}$ must also contain $q z p$ as a scattered subword. Therefore, there is an occurrence of $z$ in $\mathbf{v}$ between the first occurrence of $q$ and the last occurrence of $p$. In view of Lemma 7.3 and its dual, using an identity in $\left\{x t_{1} x t_{2} x \approx x t_{1} x x t_{2} x, x t_{1} y x t_{2} y \approx\right.$ $\left.x t_{1} x y x t_{2} y, y t_{1} x x y t_{2} x \approx y t_{1} x x y x t_{2} x\right\}^{\delta}$ one can insert an occurrence of $z$ in the block $\mathbf{B}^{\prime}$.

In view of Claim 15 we may assume that the corresponding blocks in $\mathbf{u}$ and $\mathbf{v}$ have the same contents. Now such an identity can be easily derived from $\left\{x t_{1} x t_{2} x \approx\right.$ $\left.x t_{1} x x t_{2} x\right\}^{\delta} \cup \Delta^{\delta}$.

Theorem 7.2, Proposition 4.2((i) $\rightarrow$ (iii)) and Corollary 6.4 in [13] yield an alternative proof of the following.

Corollary 7.6. [3, Theorem 3.4] The set of identities $J_{m}$ is finitely based if and only if $m \leq 3$.

\section{Acknowledgement}

The author thanks Gili Golan, Edmond Lee, Wenting Zhang and an anonymous referee for helpful comments and suggestions.

\section{References}

[1] Ashikhmin, D. N., Volkov, M. V., Zhang, W. T.: The finite basis problem for Kiselman monoids. Preprint, available under arXiv:1411.0223[math.GR]

[2] Blanchet-Sadri, F.: Equations and dot-depth one. Semigroup Forum, 47, 305317 (1993)

[3] Blanchet-Sadri, F.: Equations and monoid varieties of dot-depth one and two. Theoret. Comput. Sci. 123, 239-258 (1994)

[4] Edmunds, C.C.: On certain finitely based varieties of semigroups. Semigroup Forum, 15(1), 21-39 (1977)

[5] Eilenberg, S.: Automata, Languages and Machines Vol. B (Academic Press, 1976)

[6] Jackson, M. G.: Finiteness properties of varieties and the restriction to finite algebras. Semigroup Forum, 70, 159-187 (2005)

[7] Jackson, M. G., Sapir, O. B.: Finitely based, finite sets of words. Internat. J. Algebra Comput., 10(6), 683-708 (2000) 
[8] Lee, E. W. H.: Finitely generated limit varieties of aperiodic monoids with central idempotents. J. Algebra Appl. 8(6), 779-796 (2009)

[9] Lee, E. W. H.: Maximal Specht varieties of monoids. Mosc. Math. J. 12, 787-802 (2012)

[10] Perkins, P. : Bases for equational theories of semigroups. J. Algebra 11, 298314 (1969)

[11] Pin, J-E.: Varietes de Languages Formels (Masson, 1984)(in French); English translation Varieties of Formal Languages (North Oxford Academic, 1986 and Plenum, 1986)

[12] Sapir, O. B.: Finitely based words, Internat. J. Algebra Comput. 10(4) 457$480(2000)$

[13] Sapir, O. B.: Non-finitely based monoids, Semigroup Forum 90(3), 557-586 (2015)

[14] Sapir, O. B.: The finite basis problem for words with at most two non-linear variables. Preprint, available under arXiv:1403.6430[math.GR]

[15] Sapir, O. B.: Finitely based sets of 2-limited block-2-simple words. Preprint, available under arXiv:1509.07920[math.GR]

[16] Shevrin, L. N., Volkov, M. V.: Identities of semigroups. Russian Math (Iz. VUZ), 29(11), 1-64 (1985)

[17] Volkov, M. V.: A general finite basis condition for system of semigroup identities. Semigroup Forum, 86, 181-191 (1990)

[18] Volkov, M. V.: Reflexive relations, extensive transformations and piecewise testable languages of a given height. Internat. J. Algebra Comput. $14(5,6)$, 817-827 (2004) 